\title{
Validation of PANS and active flow control for a generic truck cabin
}

\author{
G. Minelli ${ }^{\mathrm{a}}$, E. Adi Hartono ${ }^{\mathrm{a}}$, V. Chernoray ${ }^{\mathrm{a}}$, L. Hjelm$^{\mathrm{b}}$, B. Basara ${ }^{\mathrm{c}}, \mathrm{S}$ \\ Krajnovića \\ ${ }^{a}$ Department of Mechanics and Maritime Sciences, Chalmers University of Technology, \\ Gothenburg, Sweden \\ ${ }^{b}$ Volvo Trucks AB, Gothenburg, Sweden \\ ${ }^{c}$ Advanced Simulation Technology, AVL List GmbH, Hans-List-Platz, 8020 Graz, Austria
}

\begin{abstract}
This paper presents a drag reduction study using active flow control (AFC) on a generic bluff body. The model consists of a simplified truck cabin, characterized by sharp edge separation on top and bottom edges and pressure induced separation on the two other rounded vertical front corners. The pressure induced separation reproduces the flow detachment occurring at the front A-pillar of a real truck [1]. The prediction of the flow field by partially averaged Navier-Stokes (PANS) simulations, conducted on a relatively coarse mesh, is validated against wind tunnel data (pressure measurements and particle image velocimetry (PIV)) and resolved large eddy simulations (LES) data. The Reynolds number for both simulations and experiments is $R e=$ $5 \times 10^{5}$ (which corresponds to $1 / 6$ of a full scale truck $R e$ ) based on the inlet velocity $U_{\text {inf }}$ and the width of the model $W=0.4 \mathrm{~m}$. A validation of PANS results is followed by a CFD study on the actuation frequency that minimizes the aerodynamic drag and suppresses the side recirculation bubbles. PANS accurately predicts the flow field measured in experiments and predicted by a resolved LES. The side recirculation bubble of a simplified truck cabin model is suppressed almost completely and a notable drag reduction by means of AFC is observed.
\end{abstract}

Keywords: Partially Averaged Navier-Stokes, PANS, Large Eddy Simulation, LES, Experiments, Active flow control, AFC, Proper Orthogonal Decomposition, POD, Truck, Drag reduction, Vehicle

Email address: minelli@chalmers.se (G. Minelli)

Preprint submitted to Journal of Wind Engineering and Industrial AerodynamicsAugust 25, 2017 
aerodynamics

\section{Introduction}

Heavy road vehicles present an external flow complexity defined by a turbulent boundary layer, massive separation, shear layer evolution and reattachment. All these phenomena are of great interest to aerodynamicists, world leading truck companies and truck fleet owners. In fact, the aerodynamic losses of a truck at cruise speed reach $60-70 \%$ of the total losses $[2,3,1]$. Therefore, an optimized aerodynamic design is beneficial for fuel consumption and emission reduction. Starting from the early 1970s the aerodynamic of heavy vehicles has significantly evolved, and this has enhanced their efficiency. The work presented in $[4,5,6,7,3,8]$ are just a few examples of many developments during the years. As a result, aerodynamic solutions and add-ons are extensively employed and promoted by truck fleet owners and companies, respectively.

As often happens in the aerodynamic field, the pioneering findings of aeronautical research have inspired new flow control techniques for road vehicles. Active flow control (AFC) is not an exception. Different from passive flow control techniques, AFC opens the possibility for feedback or, better put, closed-loop control [9]. Thus, an ideal AFC is not merely studied for reduction of aerodynamic drag but it could also enhance the stability of the vehicle and the ride comfort. Among the multitude of AFC techniques, a zero net mass flux (ZNMF) synthetic jet is chosen in this work as a control device. This control technique has been extensively used in different aerodynamic fields to control flow separation. It has been used to manipulate the wake of bluff bodies [10] and generic vehicles [11, 12], as well as to prevent the stall of aerofoils at high angle of attack [13, 14, 15]. Several reviews of their development and potential applications are presented by different authors $[16,17,18,19,9]$. The prevention of large scale flow separation is the ultimate and common goal of the aforementioned studies. A closer observation of the flow features of a truck shows that there are four main drag sources due to massive flow separation: the wheels and under-body, the wake, the gap between the tractor and trailer, and the front separation, Fig. 1 (a). This work focuses on the front separation occurring at the A-pillar of a truck cabin, Fig. 1 (b). This kind of separated flow can be reconnected to studies on leading-edge separations $[20,21]$. Different flow control techniques have 
been investigated to overcome this pressure induced detachment of the flow, from suction and oscillatory blowing [22, 23] to plasma actuators [24]. In this specific case, a simplified model (Fig. 2 and Tab. 1) is chosen to reproduce the A-pillar flow separation and a synthetic jet (Fig. 1 (c)) is used to control its behaviour. The working principle of this device is described in Fig. 1 (c). A flexible diaphragm in a sealed cavity generates a periodic suction and blowing of air at the opened slot at the A-pillar of the truck cabin model.

Despite recent progress in large eddy simulation (LES) and ever growing computational resources, an accurate LES calculation for detailed bluff bodies (vehicles) is still difficult to achieve, mainly because of the dense mesh resolution required. For this reason, a hybrid method, partially averaged Navier-Stokes (PANS), is used in this work. PANS was already proven to be effective for different bluff body flows [25, 26, 27], but its potential in predicting the present flow case requires further validation. In particular, PIV and surface pressure measurements were performed in the closed-circuit Chalmers University wind tunnel and used as a benchmark for the numerical validation.

This work is a continuation of a previous LES study [28] and an experimental study [29], where, an optimal actuation frequency was found to control the separation of the boundary layer from a rounded edge. The work in the present paper is a further step toward the implementation of a realistic truck A-pillar flow control, in which the following goals are achieved:

- The PANS approach is investigated and validated for the unactuated flow configuration against experiments and resolved LES.

- Pressure, velocity and Reynolds stress profiles are compared.

- Proper orthogonal decomposition (POD) and fast Fourier transform (FFT) results are used for an extended validation and are shown to be effective tools for a flow study.

- The main features of the unactuated case are described in terms of flow structures and frequencies.

- Following the POD results and the findings of two previous studies $[28,29]$, three different actuation frequencies are chosen.

- A reduction of the recirculation bubble is achieved and described for the actuated cases. 
(a)

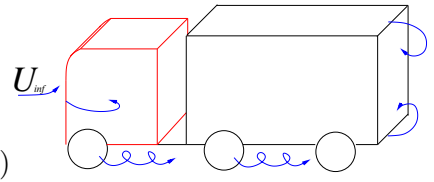

(b)

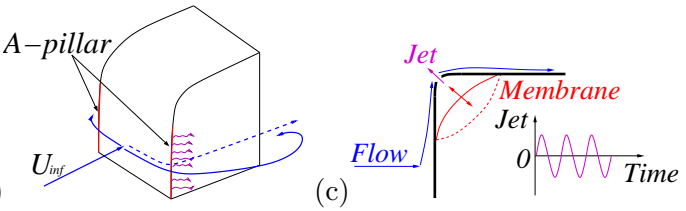

Figure 1: Main sources of aerodynamic drag for a truck (a). The A-pillar separation and the effect of the actuation (b). the solid blue line shows the unforced flow while the dashed blue line show an ideally forced flow condition. Jet flow by means of a membrane motion (c).

The remainder of the article is organised as follows: chapter 2 details the numerical formulation, the model used and the numerical and experimental set-up. Chapter 3 is divided in two main parts: first, results regarding the validation of PANS compared to resolved LES results and experimental data are presented. Second, an AFC application is simulated using the PANS equations. Conclusions are presented in chapter 4.

\section{Set-up}

The interrogated region, the numerical set-up and the experimental setup are described in this section.

\subsection{Domain and interrogated region}

The computational domain, Fig. 2 (a), was designed to reproduce the main dimensions of the wind tunnel's test section used for the experiments, Fig. 2 (b). All the dimensions are scaled by the model's width $W=0.4 \mathrm{~m}$, Tab. 1. 2D snapshots of the flow were recorded during experiments and compared to simulations. Pressure (only for simulations) and velocity data (for both simulations and experiments) were stored on a finite grid plane placed at $z=0$ (model centreline, see Fig. 2 (c) for coordinate system), Fig. 3. The window size observed in both CFD and experiments is $1 \mathrm{~W} \times 0.5 \mathrm{~W}$, as visualized in Fig. 3 (a). Snapshots from both numerical simulations and experiments were later employed for POD and FFT analyses.

\subsection{Numerical set-up}

LES and PANS were employed for the numerical study. The same boundary conditions were applied for both methods. A homogeneous Neumann boundary condition was applied at the outlet. The surfaces of the body and 


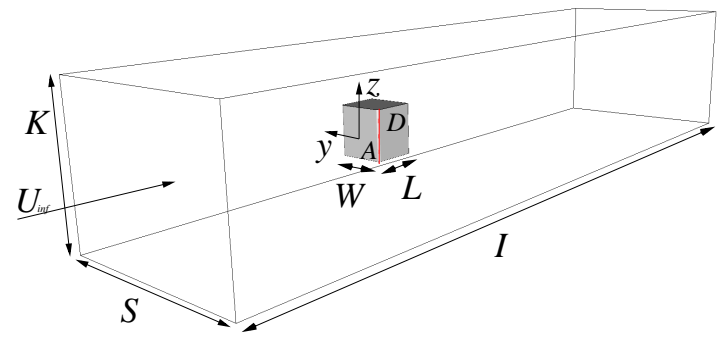

(a)

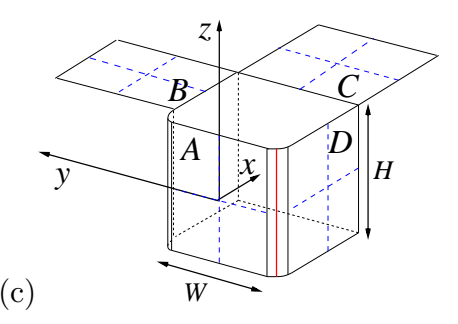

(b)

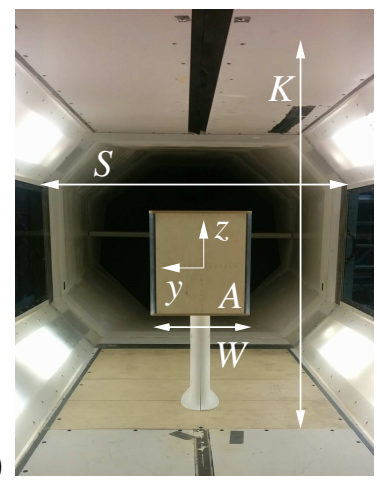

(d)

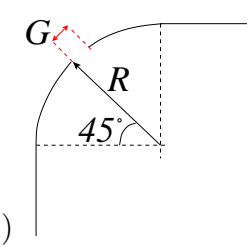

Figure 2: Computational and experimental setup (a). Wind tunnel test section and the model in place (b). A sketch of the model (c); the name of each face and the location of the pressure tap arrays (dashed blue lines). Zoom-in of the rounded corner and slot position (d). Dimensions are reported in Tab. 1.

(a)

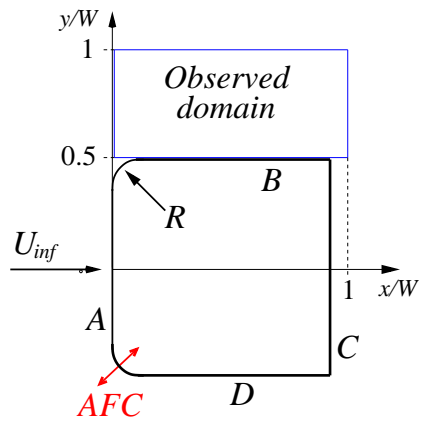

(b)

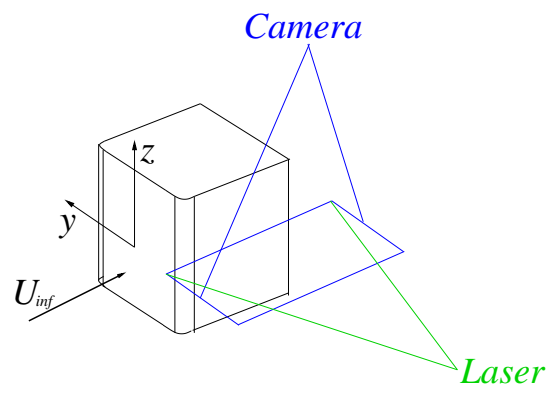

Figure 3: Observed domain dimensions (a) and a sketch of the PIV interrogated (b). 


\begin{tabular}{lllllll}
\hline$H$ & $G$ & $L$ & $I$ & $K$ & $S$ & $R$ \\
\hline 1 & 0.0025 & 0.9 & 17.5 & 3 & 4.5 & 0.05 \\
\hline
\end{tabular}

Table 1: Dimensions of the domain and the model scaled by the model width $W=0.4 \mathrm{~m}$. Letters refer to Fig. 2.

the wind tunnel walls were treated as no-slip walls. A time varying velocity (Eq. 26), reproduced the jet flow described by Fig. 1 (c). When the flow is unactuated, the AFC surface was defined as a no-slip wall, likewise the rest of the body. The position of the actuator and the slot dimension are described by Fig. 2 (d) and Tab. 1, respectively. The numerical study of the AFC aims to show the potential of such a technique, therefore, the simulations presented in section 3.2 show qualitative results of the effectiveness of this control. Future investigations and validations will be performed for a quantitative study toward a more realistic numerical modelling of the AFC boundary condition.

\subsubsection{The LES equations}

The governing LES equations are the spatially implicit filtered NavierStokes equations, where the spatial filter is determined by the characteristic width $\Delta=\left(\Delta_{1} \Delta_{2} \Delta_{3}\right)^{\frac{1}{3}}$, and $\Delta_{i}$ is the computational cell size in the three coordinate directions.

$$
\frac{\partial \bar{u}_{i}}{\partial x_{i}}=0
$$

and

$$
\frac{\partial \bar{u}_{i}}{\partial t}+\frac{\partial}{\partial x_{j}}\left(\bar{u}_{i} \bar{u}_{j}\right)=-\frac{1}{\rho} \frac{\partial \bar{p}}{\partial x_{i}}+\nu \frac{\partial^{2} \bar{u}_{i}}{\partial x_{j} \partial x_{j}}-\frac{\partial \tau_{i j}}{\partial x_{j}} .
$$

Here, $\bar{u}_{i}$ and $\bar{p}_{i}$ are the resolved velocity and pressure, respectively, and the bars over the variables denote the operation of filtering. The influence of the small scales in equation 2 appears in the SGS stress tensor, $\tau_{i j}=\overline{u_{i} u_{j}}-\bar{u}_{i} \bar{u}_{j}$. The algebraic eddy viscosity model, described in [30], was employed in this work. The Smagorinsky model represents the anisotropic part of the SGS stress tensor, $\tau_{i j}$ as

$$
\tau_{i j}-\frac{1}{3} \delta_{i j} \tau_{k k}=-2 \nu_{s g s} \bar{S}_{i j}
$$

where the SGS viscosity,

$$
\nu_{s g s}=\left(C_{s} f_{v d} \Delta\right)^{2}|\bar{S}|
$$


116

117

and,

$$
\bar{S}=\sqrt{\left(2 \bar{S}_{i j} \bar{S}_{i j}\right)}
$$

where

$$
\bar{S}_{i j}=\frac{1}{2}\left(\frac{\partial \bar{u}_{i}}{\partial x_{j}}+\frac{\partial \bar{u}_{j}}{\partial x_{i}}\right) .
$$

The Smagorinsky constant, $C_{s}=0.1$, previously used in bluff body LES [31], is used in the present work. $f_{v d}$, in equation 4 , is the Van Driest damping function,

$$
f_{v d}=1-\exp \left(\frac{-n^{+}}{25}\right)
$$

where $n^{+}$is the wall normal distance in viscous units.

\subsubsection{The PANS equations}

The PANS governing equations are defined by the following model $[32,33]$.

$$
\frac{\partial U_{i}}{\partial x}=0
$$

$$
\frac{\partial U_{i}}{\partial t}+U_{j} \frac{\partial U_{i}}{\partial x_{j}}=-\frac{1}{\rho} \frac{\partial p}{\partial x_{i}}+\frac{\partial}{\partial x_{j}}\left(\nu \frac{\partial U_{i}}{\partial x_{j}}-\tau\left(V_{i}, V_{j}\right)\right)
$$

where $\tau\left(V_{i}, V_{j}\right)$ is the generalized second moment [34] and represents the effect of the unresolved scales on the resolved field. The Boussinesq assumption is now invoked to model the second moment:

$$
\tau\left(V_{i}, V_{j}\right)=-2 \nu_{u} S_{i j}+\frac{2}{3} k_{u} \delta_{i j} .
$$

Here, $k_{u}$ is the unresolved kinetic energy, $S_{i j}=\frac{1}{2}\left(\partial U_{i} / \partial x_{j}+\partial U_{j} / \partial x_{i}\right)$ is the resolved stress tensor, and $\nu_{u}=C_{\mu} \zeta_{u} k_{u}^{2} / \varepsilon_{u}$ is the viscosity of the unresolved scales where $\zeta=\overline{v_{u}^{2}} / k_{u}$ is the velocity scale ratio of the unresolved velocity scale $\overline{v_{u}^{2}}$ and unresolved turbulent kinetic energy $k_{u} \cdot \overline{v_{u}^{2}}$ refers to the normal fluctuating component of the velocity field to any no-slip boundary. At this stage, three transport equations for $k_{u}-\varepsilon_{u}-\zeta_{u}$ and a Poisson equation for the elliptic relaxation function of the unresolved velocity scales are necessary to close the model. Thus, the complete PANS $k-\varepsilon-\zeta-f$ model is given by the following set of equations:

$$
\frac{\partial k_{u}}{\partial t}+U_{j} \frac{\partial k_{u}}{\partial x_{j}}=P_{u}-\varepsilon_{u}+\frac{\nu_{u}}{\sigma_{k_{u}}} \frac{\partial^{2} k_{u}}{\partial x_{j}^{2}}
$$


137

138

139

14

152

$$
\frac{\partial \varepsilon_{u}}{\partial t}+U_{j} \frac{\partial \varepsilon_{u}}{\partial x_{j}}=C_{\varepsilon 1} P_{u} \frac{\varepsilon_{u}}{k_{u}}-C_{\varepsilon 2}^{*} \frac{\varepsilon_{u}^{2}}{k_{u}}+\frac{\nu_{u}}{\sigma_{\varepsilon_{u}}} \frac{\partial^{2} \varepsilon_{u}}{\partial x_{j}^{2}}
$$

$$
\frac{\partial \zeta_{u}}{\partial t}+U_{j} \frac{\partial \zeta_{u}}{\partial x_{j}}=f_{u}-\frac{\zeta_{u}}{k_{u}}\left(\varepsilon_{u}\left(1-f_{k}\right)-P_{u}\right)+\frac{\nu_{u}}{\sigma_{\zeta_{u}}} \frac{\partial^{2} \zeta_{u}}{\partial x_{j}^{2}}
$$

$$
L_{u}^{2} \nabla^{2} f_{u}-f_{u}=\frac{1}{T_{u}}\left(c_{1}+c_{2} \frac{P_{u}}{\varepsilon_{u}}\right)\left(\zeta_{u}-\frac{2}{3}\right) .
$$

$\nu_{u}=C_{\mu} \zeta_{u} \frac{k_{u}^{2}}{\varepsilon_{u}}$ is the unresolved turbulent viscosity. $P_{u}=-\tau\left(V_{i}, V_{j}\right) \frac{\partial U_{i}}{\partial x_{j}}$ is the production of the unresolved turbulent kinetic energy, which is closed by the Boussinesq assumption, equation 10 . The coefficients $C_{\varepsilon 2}^{*}$ and $C_{\varepsilon 1}$ are defined as:

$$
C_{\varepsilon 2}^{*}=C_{\varepsilon 1}+f_{k}\left(C_{\varepsilon 2}-C_{\varepsilon 1}\right)
$$

$$
C_{\varepsilon 1}=1.4\left(1+\frac{0.045}{\sqrt{\zeta_{u}}}\right) \text {. }
$$

$\sigma_{k_{u}}=\sigma_{k} \frac{f_{k}^{2}}{f_{\varepsilon}}$ and $\sigma_{\varepsilon_{u}}=\sigma_{\varepsilon} \frac{f_{k}^{2}}{f_{\varepsilon}}$ are the counterpart of the unresolved kinetic energy and dissipation, respectively. In this way, $f_{k}$ and $f_{\varepsilon}$ contribute to changing the turbulent transport Prandtl number contributing to the decrease of the unresolved eddy viscosity [35]. The constants appearing in equations 11 to 14 are: $C_{\mu}=0.22, C_{\varepsilon 2}=1.9, c_{1}=0.4, c_{2}=0.65, \sigma_{k}=1$, $\sigma_{\varepsilon}=1.3, \sigma_{\zeta_{u}}=1.2 . L_{u}$ and $T_{u}$ are the length and time scales defined by using the unresolved kinetic energy:

$$
L_{u}=C_{L} \max \left[\frac{k_{u}^{3 / 2}}{\varepsilon}, C_{\delta}\left(\frac{\nu^{3}}{\varepsilon}\right)^{1 / 4}\right]
$$

$$
T_{u}=\max \left[\frac{k_{u}}{\varepsilon}, C_{\tau}\left(\frac{\nu}{\varepsilon}\right)^{1 / 2}\right]
$$

where $C_{\tau}=6, C_{L}=0.36$ and $C_{\delta}=85$. A more detailed explanation of the construction of the equations is given in $[36,37] . f_{k}$ and $f_{\varepsilon}$ are the ratios between resolved to total kinetic energy and dissipation, respectively and they are the key factors that make the model act dynamically. They can assume values between 1 and 0 according to the selected cut-off. The dynamic parameter was proposed as the ratio between the geometric averaged 


\begin{tabular}{lllllllll}
\hline Grid & Size & $n_{\text {mean }}^{+}$ & $n_{\max }^{+}$ & $\Delta l_{\max }^{+}$ & $\Delta s_{\max }^{+}$ & $\Delta l_{\text {mean }}^{+}$ & $\Delta s_{\text {mean }}^{+}$ & $\mathrm{CFL}$ \\
\hline Fine & 16 mil. & $<0.5$ & $<2$ & $<100$ & $<100$ & $<35$ & $<35$ & $<1$ \\
Coarse & 4 mil. & $<0.5$ & $<2$ & $<450$ & $<450$ & $<120$ & $<120$ & $<1$ \\
\hline
\end{tabular}

Table 2: Details of the computational grids.

grid cell dimension, $\Delta=\left(\Delta_{x} \Delta_{y} \Delta_{z}\right)^{1 / 3}$, and the Taylor scale of turbulence, $\Lambda=\frac{\left(k_{u}+k_{r e s}\right)^{3 / 2}}{\varepsilon}[38]$ :

$$
f_{k}(x, t)=\frac{1}{\sqrt{C_{\mu}}}\left(\frac{\Delta}{\Lambda}\right)^{2 / 3} .
$$

\subsubsection{The mesh resolution}

The simulations in this study were performed with the commercial finite volume CFD solver, AVL FIRE [39]. AVL FIRE is based on the cell-centred finite volume approach. The grid topology was constructed using the Ogrid technique in order to concentrate most of the computational cells close to the body. Figure 4 shows the discretization of the model's surface of the coarse and the fine grid. A reliable LES grid should resolve $80 \%$ of the turbulent energy [40]. According to [41], the first grid point in the wall normal direction must be located at $n^{+}<1$, where $n^{+}=\frac{u_{\tau} n}{\nu}$ with the friction velocity $u_{\tau}$. The resolution in the span-wise and stream-wise directions must be $\Delta l^{+} \simeq 15-40$ and $\Delta s^{+} \simeq 50-150$, respectively, in order to resolve the near-wall structures. Here $\Delta l^{+}=\frac{u_{\tau} \Delta l}{\nu}$ and $\Delta s^{+}=\frac{u_{\tau} \Delta s}{\nu}$. The grid resolution of the two grids employed is described in Tab. 2 and visualized in Fig. 4. In particular, $n_{\text {mean }}^{+}$and the CFL number were under 1 all over the surface of the model and in the flow domain, respectively. Only few elements at the sharp top and bottom edges of the model gives $n^{+}$values larger than 1 but anyway lower than 2 .

\subsection{Experimental set-up}

Experiments were carried out in the closed circuit wind tunnel at Chalmers University of Technology. The test section has a length of $3 \mathrm{~m}$, a width of $1.8 \mathrm{~m}$ and a height of $1.25 \mathrm{~m}$ with a stable speed up to $60 \mathrm{~m} / \mathrm{s}$. The flow turbulence level was within $0.15 \%$ at a frequency range between $1 \mathrm{~Hz}$ and $10000 \mathrm{~Hz}$. Shown in Fig. 2 (b), is the model placed in the wind tunnel's test section. Two vertical stripes of coarse randomly distributed roughness were placed on the frontal surface (face A Fig. 2 (c)) to ensure turbulence transition 

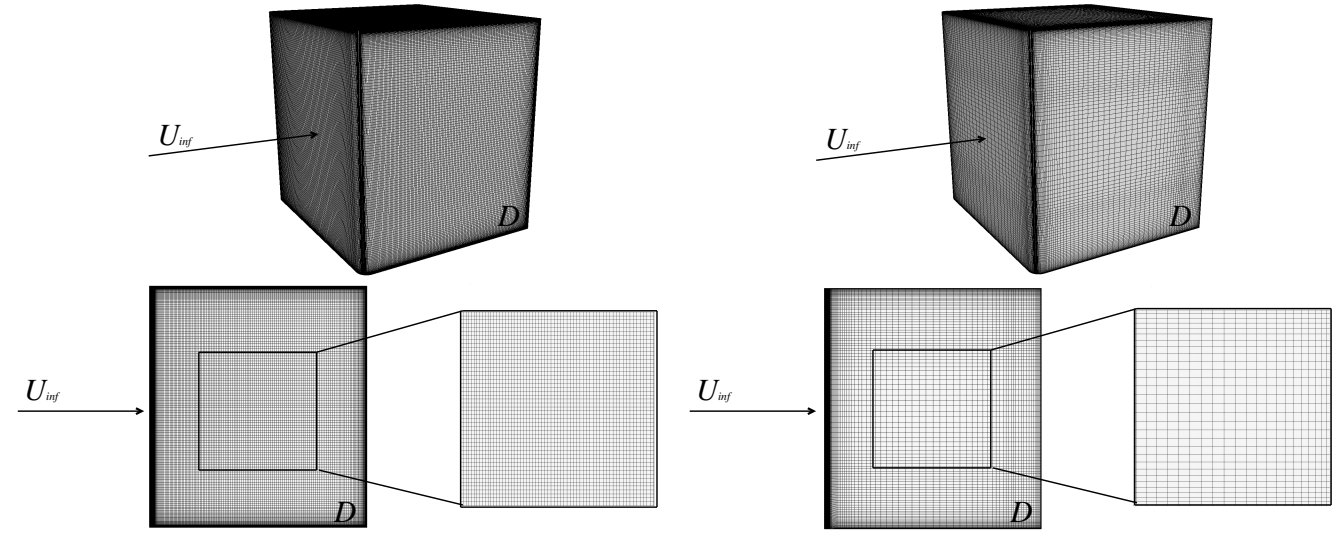

Figure 4: Fine (left) and coarse (right) surface grids visualization.

and trip the boundary layer in all experimental runs. The support of the model was profiled by a NACA profile to avoid vortex shedding, ensure the vertical symmetry of the flow and save computational efforts. In fact, the support was not simulated in numerics and the model was represented by a suspended body, keeping the same ground clearance of the experiments. The experimental model was equipped with horizontal and vertical arrays of pressure taps (placed along the dashed blue lines in Fig. 2 (c)) for evaluation of the coefficient of pressure $C_{p}$. The front $(A)$, windward $(B)$, leeward $(D)$ and base $(C)$ faces are shown in Fig. 2 (c). For simplicity, faces $B$ and $D$ are termed windward and leeward, respectively, also in the 0 yaw angle $(\beta=0)$ configuration. The pressure data were obtained using two 48-channel Scanivalve systems (NetScanner ${ }^{T M}$ model 9116). The pressure system has an accuracy of $\pm 0.2 \mathrm{~Pa}$ for the used pressure range $( \pm 300 \mathrm{~Pa})$. The pressure signals were time averaged over a period of $2 \mathrm{~s}$. Only the time averaged pressure values are used for the comparison with the CFD results.

PIV images were recorded by a monochrome double-frame SCMOS camera SpeedSense M340 by Dantec with a 2560 pixels by 1600 pixels resolution, 12 bit pixel depth, and $10 \mu \mathrm{m}$ pixel size. The camera was equipped with a 105-mm f/2.8 lens from Sigma. The camera registered image pairs at a $400 \mathrm{~Hz}$ frame rate at full resolution in double frame mode (with a time between pulses of $60 \mu \mathrm{s})$. Flow seeding was achieved with a fog generator and glycol-based fluid. The Dual Power Nd:YLF LDY300-PIV laser from Litron provided up to $2 \times 30 \mathrm{~mJ}$ at $1000 \mathrm{~Hz}$ and a $527 \mathrm{~nm}$ wavelength. The laser 
was equipped with a laser guiding arm and laser sheet optics. The flow field area illuminated was $200 \times 400 \mathrm{~mm}^{2}$. Dantec Dynamic Studio 2015 software was used for data acquisition and post-processing. Each data set includes 800 images, which corresponds to a measurement period of 2 seconds with a spatial resolution of $0.125 \times 0.156 \mathrm{~mm}^{2}$ per pixel. The vector calculation is performed in multi-pass procedure with a decreasing window size. The initial interrogation window size is 64 pixels $\times 64$ pixels with a $50 \%$ overlap and square 1:1 weighing factor for the first two passes. Finally, three passes are performed with a 32 pixels $\times 32$ pixels window size, $50 \%$ overlap and round 1:1 Gaussian weighing factor. The velocity uncertainty was estimated as $0.1 \mathrm{~m} / \mathrm{s}$ for the time averaged velocity.

\subsection{Modal and frequency analyses}

An FFT analysis highlights the spatial area of interest and the energy level of a certain frequencies in the interrogated flow field. It is interesting to compare this approach with POD modes in order to gain a better understanding of the flow structures in terms of both the energy content and characteristic frequencies.

The POD here is made on velocity components and pressure snapshots sampled with a constant time step. The span-wise $(y)$ velocity component (the same approach can be applied to the relative pressure variable) set of snapshots is described by $v^{m}=v\left(\boldsymbol{x}, t^{m}\right)$ at time $t^{m}=m \Delta t, m=1, \ldots, M$ with the time $\Delta t$, and a Cartesian coordinate system $\boldsymbol{x}=(x, y)$ with unit vectors $\boldsymbol{e}_{x}, \boldsymbol{e}_{y}$ respectively.

As was originally proposed in [42] and later introduced with the method of snapshots in [43], this method is based on energy ranking of orthogonal structures computed from a correlation matrix of the snapshots. A singular value decomposition (SVD) approach is used to conduct the POD analysis on the set of snapshots mentioned. Note that the snapshot POD method limits the number of POD modes to $M-1$. In the present POD analysis, the wall normal velocity component is decomposed into the mean field, $\langle v\rangle$, and the fluctuating part, $v^{\prime}$, as

$$
v(\boldsymbol{x}, t)=\langle v\rangle(\boldsymbol{x})+v^{\prime}(\boldsymbol{x}, t)
$$

The fluctuating part is then approximated, by the SVD approach, with spa- 
tially dependent modes, $v_{i}$, and time dependent mode coefficient, $b_{i}$, as

$$
v^{\prime}(\boldsymbol{x}, t)=\sum_{i=1}^{\infty} b_{i}(t) v_{i}(\boldsymbol{x}) \approx \sum_{i=1}^{M-1} b_{i}(t) v_{i}(\boldsymbol{x})+v_{r e s}(\boldsymbol{x}, t)
$$

The definition can now be written in a more compact form if one considers that $b_{0}=1$ and $v_{0}=\langle v\rangle$, following [44],

$$
v(\boldsymbol{x}, t)=\sum_{i=0}^{M-1} b_{i}(t) v_{i}(\boldsymbol{x}) .
$$

The first and second moments of the POD modes coefficients are:

$$
\left\langle b_{i}\right\rangle=0 ; \quad\left\langle b_{i} b_{j}\right\rangle=\mu_{i} \delta_{i j} .
$$

The energy content of the single mode, $K_{i}$, is approximated from the mode coefficients as

$$
K_{i}(t)=\frac{1}{2} b_{i}^{2}(t)
$$

and the total energy, $K_{\Sigma}(t)$, is evaluated as

$$
K_{\Sigma}(t)=\sum_{i=1}^{M-1} K_{i}(t)
$$

In the present study, the POD analysis was performed over 800 snapshots for both CFD and PIV data. In the POD formulation, mode 1 represents the mean value of the flow field. The non-dimensional time step $\Delta t^{\star}$ between each CFD snapshot was $\Delta t_{C F D}^{\star}=\Delta t U_{\text {inf }} / W=1.92 \times 10^{-2}$. Considering the PIV snapshots, the limitations of the camera frame rate leads to a nondimensional time step between snapshots equal to $\Delta t_{E X P}^{\star}=\Delta t U_{\text {inf }} / W=$ $1.2 \times 10^{-1}$. Thus, the highest frequency considered in the modal analysis (according to the Nyquist frequency) is 200hz. On the other hand, the lowest frequency captured is limited by the maximum simulation time to $5 \mathrm{~Hz}$ (LES and PANS simulations are averaged over 1s). Concerning the FFT analysis, a classical approach is applied to the set of snapshots. The discrete time signal of each grid point of the planar snapshot is transformed into its discrete frequency domain. In this way the energy content of each frequency can be plotted, for each grid point, on the 2D domain. Figures like 11 and 14 show the energy content of a chosen frequency in each point of the domain. 


\subsection{Actuation's parameters}

The magnitude of the velocity at the actuation region $(G$ in Fig. $2(d))$, $U_{a f c}$, was defined by a time varying (uniform in space) boundary condition as follows,

$$
U_{\text {afc }}=0.26 U_{\text {inf }} \sin \left(t 2 \pi f_{a}\right),
$$

where $U_{i n f}$ is the magnitude of the free stream velocity, and $f_{a}$ is the actuation frequency. A simple uniformity in space was chosen at this stage for a qualitative AFC application. At a later stage of the project, the uniformity will be also quantified experimentally. Two non-dimensional parameters describe the performance of the actuation. The first parameter is the momentum coefficient $C_{\eta}$, which is an indicator of the energy spent for the actuation $\left(\bar{I}_{j}\right)$ with respect to the energy of the unactuated flow.

$$
\bar{I}_{j}=\left(\frac{2}{T}\right) \rho_{j} G \int_{0}^{T / 2} U_{a f c}^{2}(t) d t
$$

$$
C_{\eta}=\frac{\bar{I}_{j}}{\frac{1}{2} \rho W U_{\text {inf }}^{2}} .
$$

Here, $\rho_{j}=\rho$ is the flow density and $T$ is the actuation period. $C_{\eta}=1.22 \times$ $10^{-4}$ is low but sufficient to excite the thin boundary layer that characterizes the attached flow upstream of separation. All the frequencies in the present work are described in terms of the second non-dimensional parameter, the reduced frequency $F^{+}$(also called actuation Strouhal number).

$$
F^{+}=\frac{f}{U_{\text {inf }} / W}
$$

Here $f$ represents the frequency in hertz.

\section{Results}

This section is divided into two parts. First, a validation of PANS against resolved LES and experimental data is presented. In particular the validation consists in the following comparisons: surface pressure profiles, velocity and Reynolds stress. POD and FFT analysis of the span-wise velocity component are used to compare PANS, LES results and experimental measurements while the POD and FFT analysis of the pressure field and the $C_{d}$ signals are used to compare PANS and LES results. In the second part of the chapter, PANS simulations are used to investigate the qualitative effects of the actuation on the aerodynamic performance of the model. 

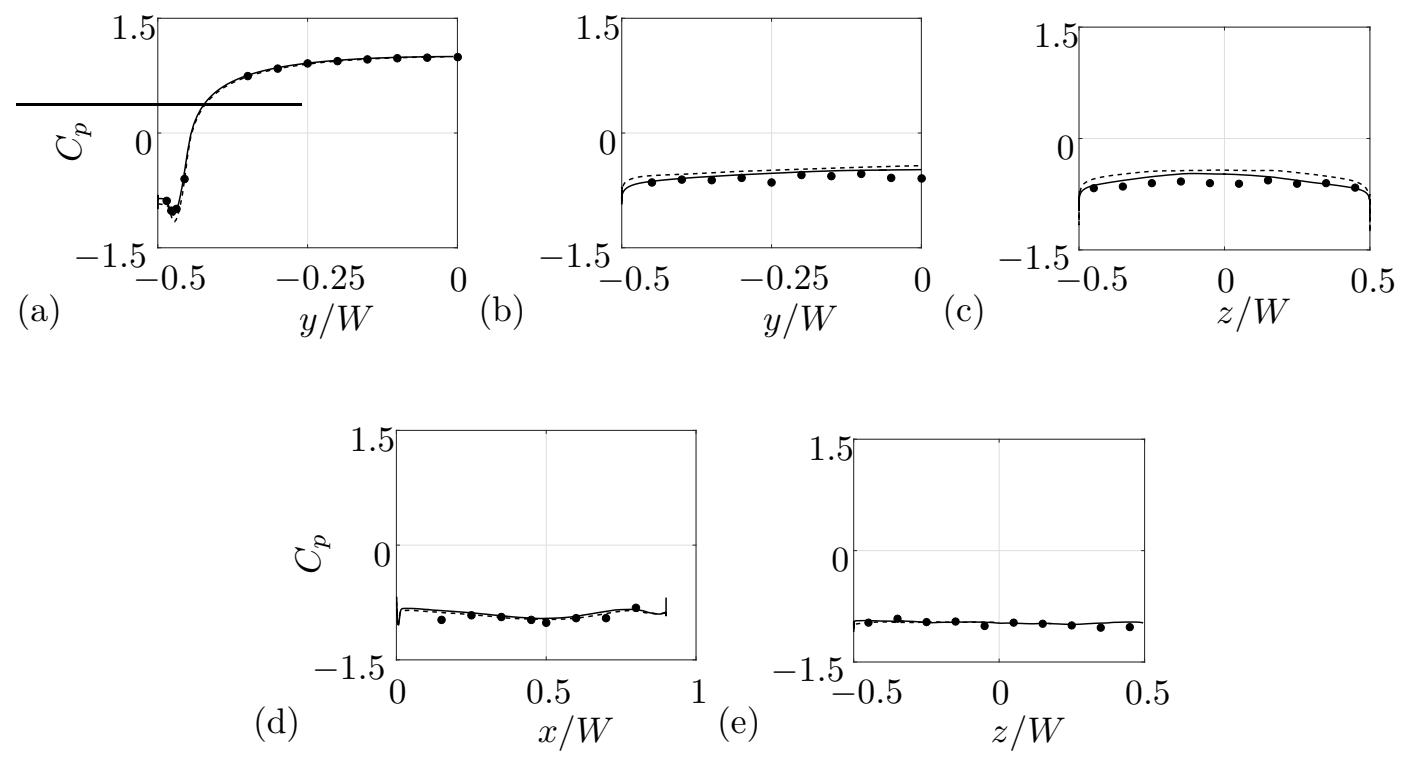

Figure 5: Comparison of $C_{p}$ profiles, $\beta=0^{\circ}$. Resolved LES (solid black line), coarse PANS (dashed black line), experiments (dots). Front, horizontal profile (a). Base, horizontal profile (b). Base, vertical profile (c). Leeward side, horizontal profile (d). Leeward side, vertical profile (e). $R e=5 \times 10^{5}$.

\subsection{Validation: PANS and LES compared to Experiments}

The goal of this validation effort is to compare the prediction capacity of PANS for a massively separated turbulent flow field. In particular, surface pressure profiles, velocity and Reynolds stress profiles, and modal analysis results are presented and compared in the following sections.

\subsubsection{Surface pressure profiles (PANS, LES and experiments)}

Pressure profiles of two configurations at yaw angles $\beta=0^{\circ}$ and $\beta=10^{\circ}$ were measured and compared with numerical simulations. PANS results obtained from the coarse grid calculation are compared with LES results obtained from the fine grid simulation and experimental data. Both the first $\left(\beta=0^{\circ}\right.$, Fig. 5) and the second $\left(\beta=10^{\circ}\right.$, Fig. 6) configurations give good agreement between experiments and simulations. The mesh employed for PANS is relatively coarse for the Reynolds number considered here, Tab. 2, and is far from being sufficient for a well resolved LES. 

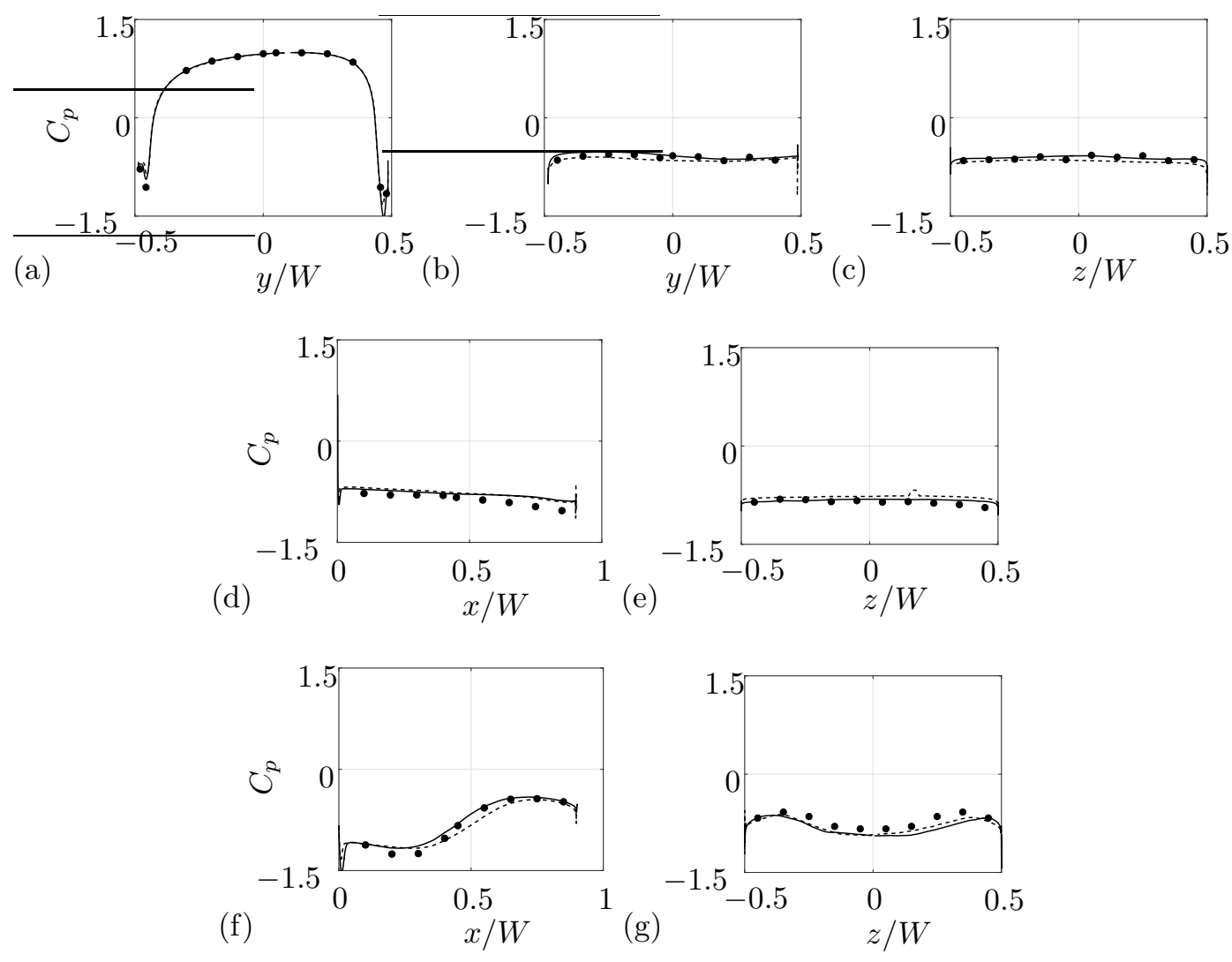

Figure 6: Comparison of $C_{p}$ profiles, $\beta=10^{\circ}$. Resolved LES (solid black line), coarse PANS (dashed black line), experiments (dots). Front, horizontal profile (a). Base, horizontal profile (b). Base, vertical profile (c). Leeward side, horizontal profile (d). Leeward side, vertical profile (e). Windward side (f), horizontal profile. Windward side, vertical profile (g). $R e=5 \times 10^{5}$. 


\subsubsection{Velocity and Reynolds stress profiles (PANS, LES and experiments)}

A 2D representation of the measured recirculation bubble and its CFD prediction, is shown in Fig. 7.

LES mispredicts the recirculation bubble when the grid is too coarse. On the other hand, PANS provides a good prediction using the same coarse mesh. This is valid for both the stream-wise (Fig. 7 (a)) and span-wise (Fig. 7 (b)) component of the velocity. The location of the side vortex is also affected by the mesh resolution and the method used. In particular, the coordinates of the coarse PANS core vortex differs by $6 \%$ and $1 \%$ (in $x$ and $y$ direction respectively) from the PIV measurements. The coarse LES vortex on the other hand, is located $30 \%$ and $9 \%$ (in $x$ and $y$ direction respectively) off from the vortex observed in PIV, while the fine LES vortex is displaced $2.5 \%$ and $0.6 \%$ (in $x$ and $y$ direction respectively) from the PIV one. As a consequence, the normal (Fig. 7 (c)) and the shear (Fig. 7 (d)) stress are also better predicted by PANS, when compared to the results of the coarse LES calculation. Figures 8 and 9 show the gap between an acceptable PANS prediction (black dashed line) and a poor LES prediction (gray solid line) calculated on the same coarse mesh. Only when the grid is fine enough is LES (black solid line) able to predict the flow with high accuracy.

\subsubsection{POD and FFT analyses of the span-wise velocity field (PANS, LES and experiments)}

The comparisons described by Figs. 10 and 11 show the capacity of PANS to predict the main flow structures and frequencies, even when a coarse grid is employed. The second and the third span-wise velocity POD modes visualize the same structures for both simulations and experiments, Fig. 10. The FFT analysis, conducted on the same set of snapshots, indicates a similar spatial distributions of the energy of the most important frequencies of the spanwise velocity component when PANS results are compared with resolved LES results and experiments, Fig. 11. Moreover, the spatial distributions of $F^{+}=0.7$ and $F^{+}=2$ (Fig. 11) match with the spatial distributions of the structures defined by modes 2 and 3 (Fig. 10). By this comparison, a dominant frequency of a coherent structure described by a POD mode can be related to the frequency highlighted by the FFT analysis.

\subsubsection{POD and FFT analyses of the pressure field (PANS and LES)}

After a first comparison with experimental data, the numerical results are deeper investigated. Flow structures observations and the results of a POD 

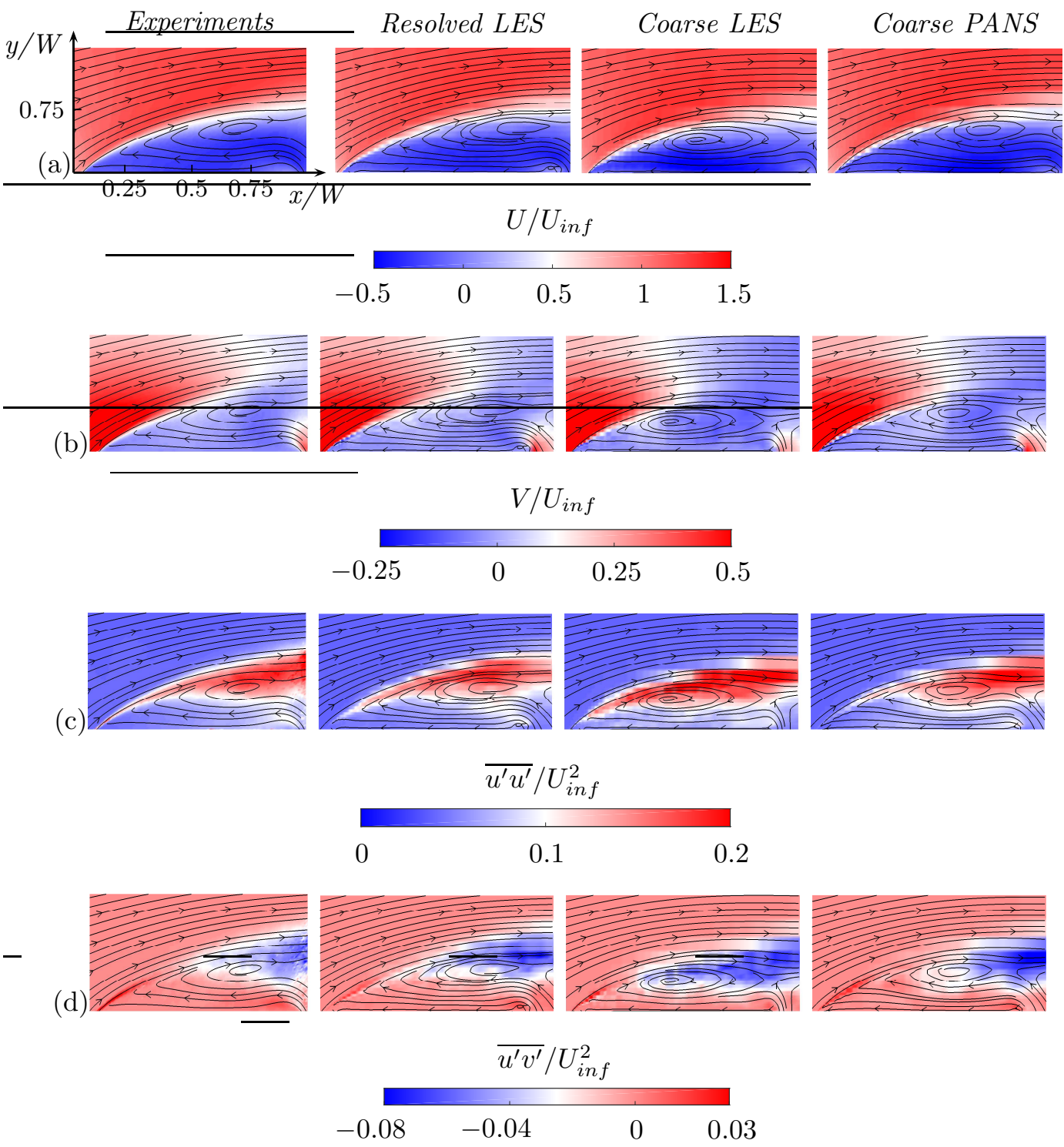

Figure 7: Averaged stream-wise (a) and span-wise ( $y$ direction) (b) velocity components, $\overline{u^{\prime} u^{\prime}}$ normal stress (c) and $\overline{u^{\prime} v^{\prime}}$ shear stress (d). From left to right: experiments, resolved LES, coarse LES, coarse PANS. Refer to Fig. 3 (a) for the observed domain location. $R e=5 \times 10^{5}$. 


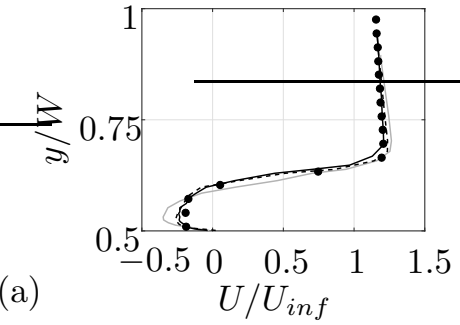

(b)

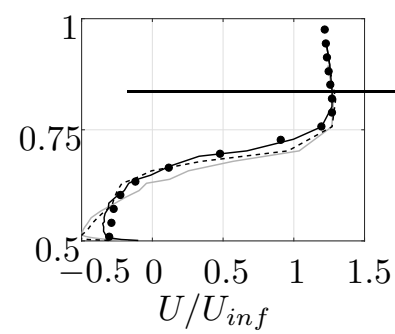

(c)
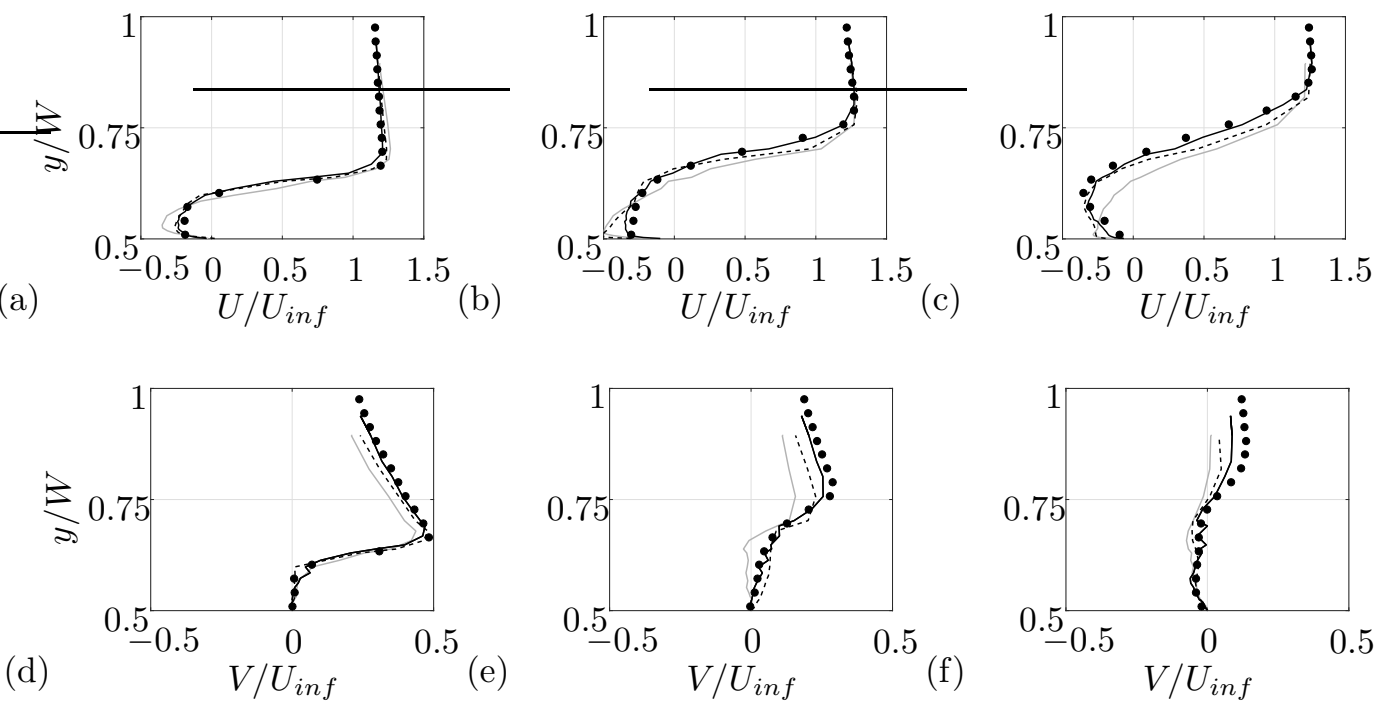

(e)
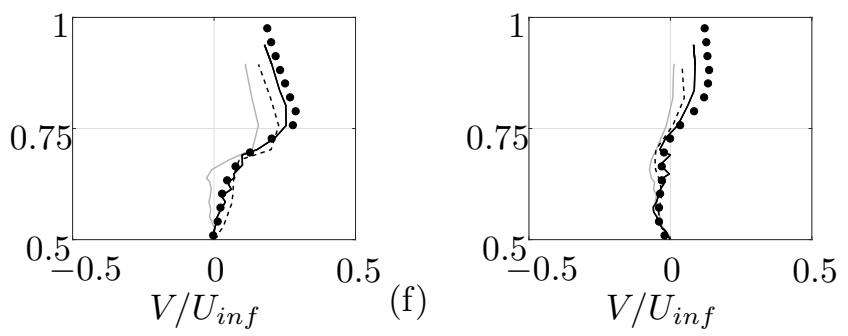

Figure 8: Averaged stream-wise (a-c) and span-wise ( $y$ direction) (d-e) velocity components at different locations along the recirculation bubble: $x_{1} / W=0.250$ (a and $\mathrm{d}$ ), $x_{2} / W=0.500$ (b and e), $x_{3} / W=0.750$ (c and f). Resolved LES (solid black line), coarse LES (solid gray line), coarse PANS (dashed black line), experiments (dots).

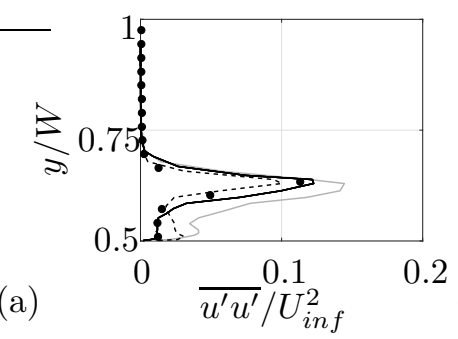

(b)
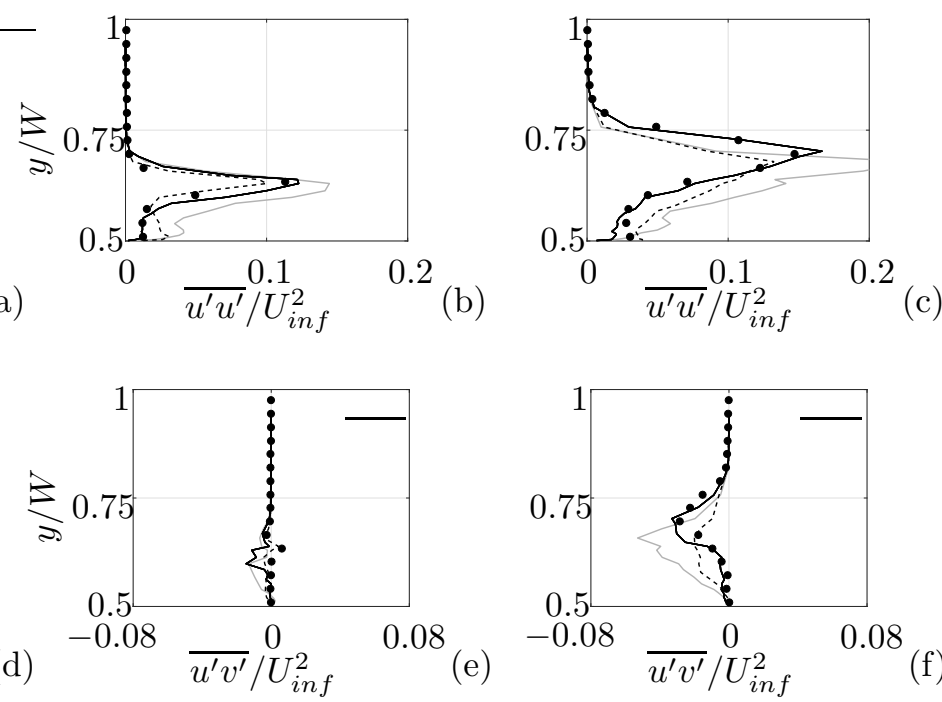

(e)

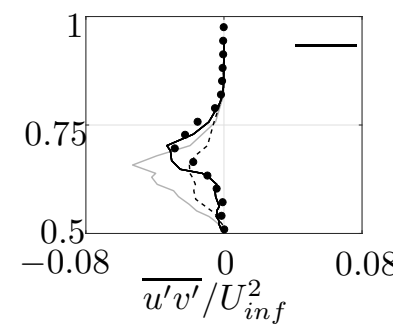

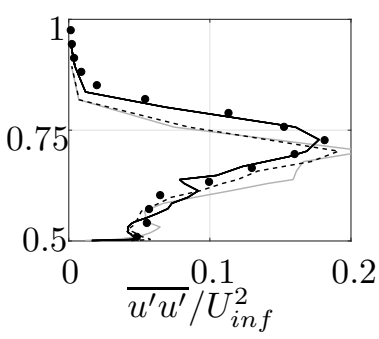

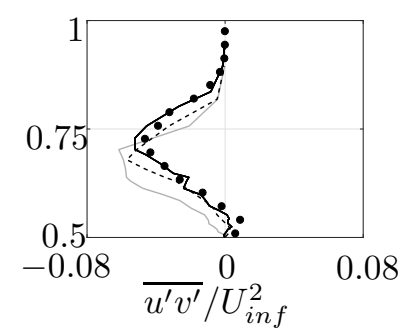

Figure 9: $\overline{u^{\prime} u^{\prime}}$ normal stress (a-c) and $\overline{u^{\prime} v^{\prime}}$ shear stress (d-e) at different locations along the recirculation bubble: $x_{1} / W=0.250$ (a and d), $x_{2} / W=0.500$ (b and e), $x_{3} / W=0.750$ (c and f). Resolved LES (solid black line), coarse LES (solid gray line), coarse PANS (dashed black line), experiments (dots). 

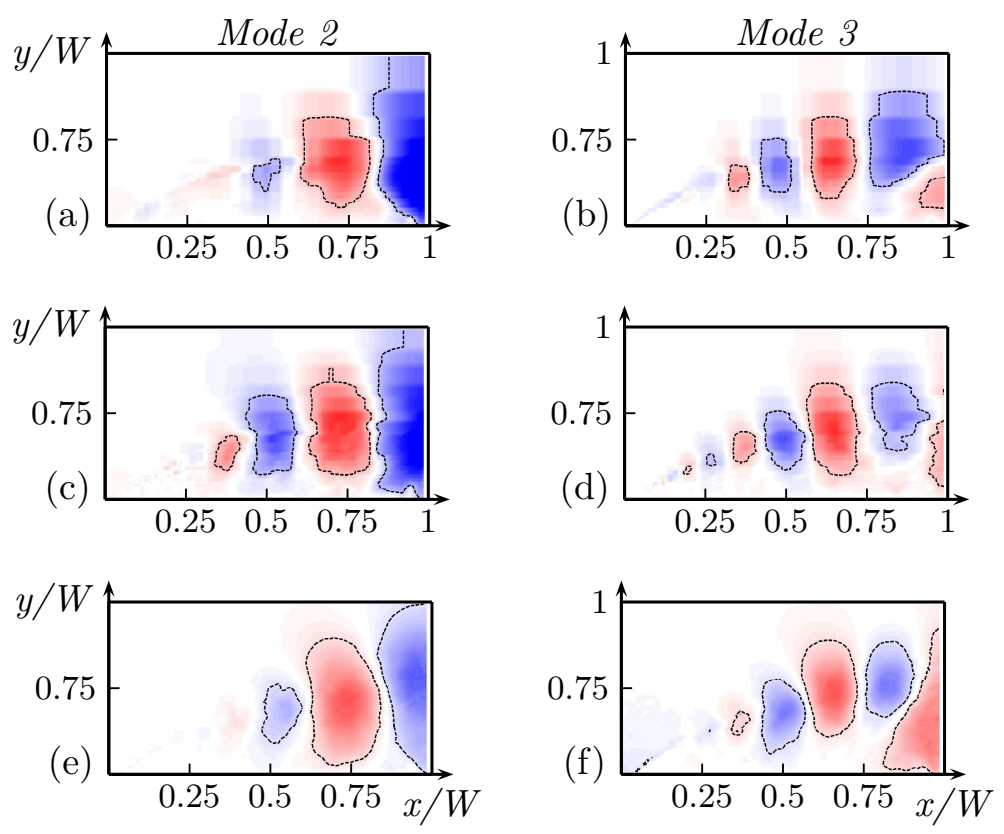

Figure 10: Span-wise velocity component ( $y$ direction) POD modes. Comparison between coarse PANS (a-b), resolved LES (c-d) and PIV (e-f) results. Refer to Fig. 3 (a) for the observed domain location. 

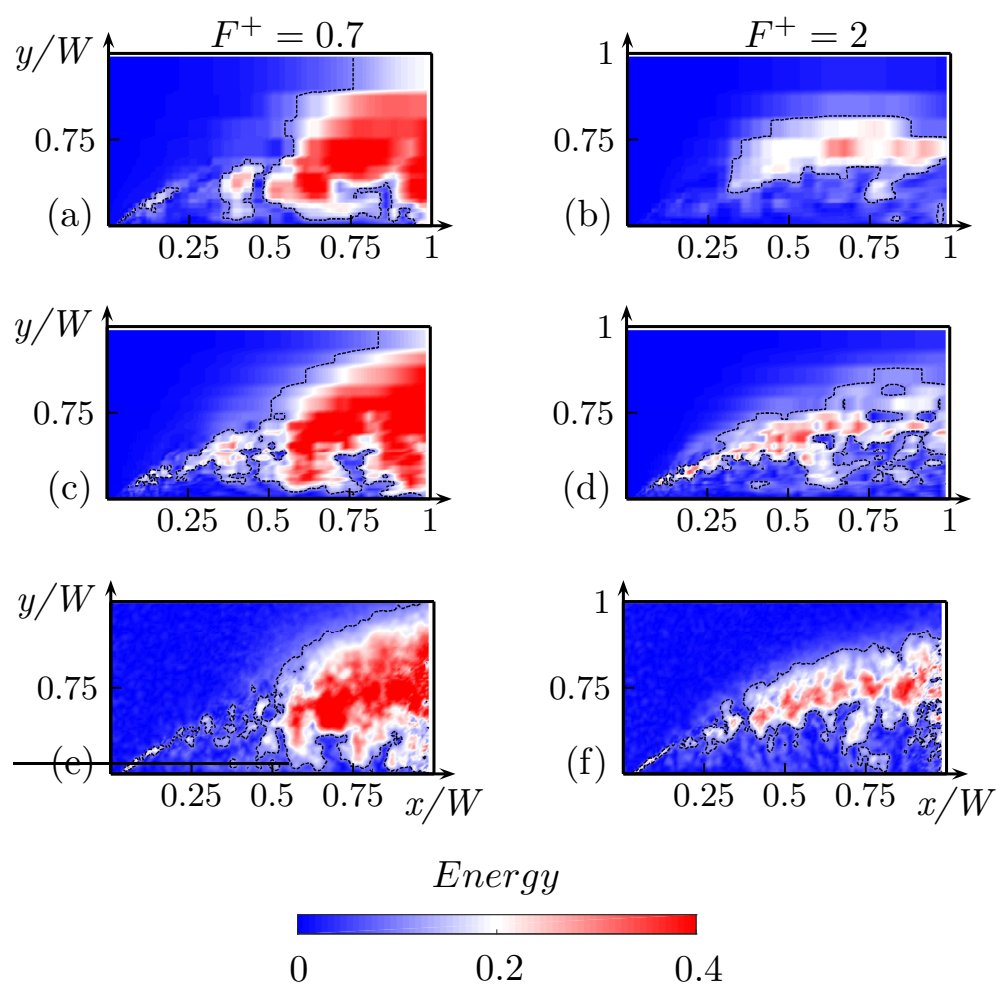

Figure 11: Spatial distribution of the energy of the characteristic frequencies of the spanwise velocity component ( $y$ direction). The values are normalized by the maximum value of the spatially averaged spectrum. Comparison between coarse PANS (a-b), resolved LES (c-d) and PIV (e-f) results. Refer to Fig. 3 (a) for the observed domain location. 


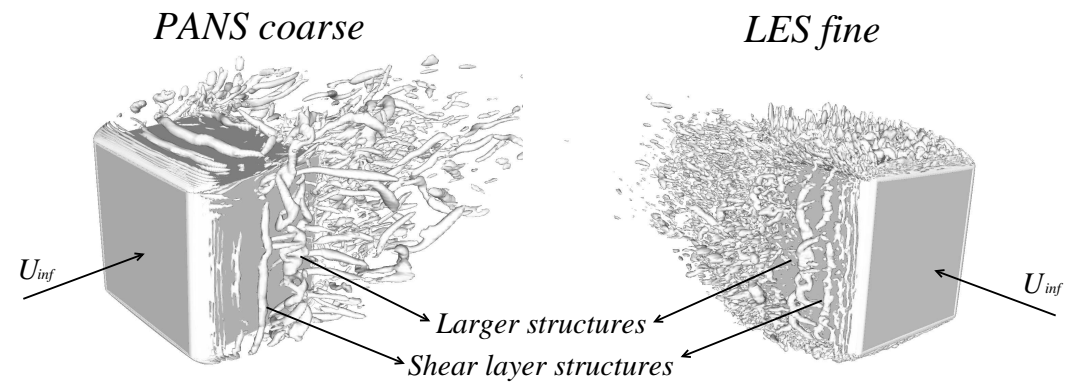

Figure 12: Isosurfaces of $Q$-criterion $\left(Q=1.5 \times 10^{5} s^{-2}\right)$. Coarse PANS (left) and resolved LES (right).

analysis of the pressure field are reported for a better understanding of the main flow features. Therefore, the PANS prediction is further investigated and compared with the resolved LES simulation. Figure 12 shows the isosurfaces of the second invariant of the velocity gradient ( $Q$-criterion) for the two methods. The resolved LES is capable of resolving smaller eddies. Nevertheless, the coarse PANS is able to capture the main flow structures. In fact, the separation mechanism and the evolution of the shear layer from small to larger eddies is well captured. Figures 13 and 14 show the prediction of the first three most energetic pressure POD modes. The prediction by resolved LES and coarse PANS is similar, and the spatial distributions of the energy of the characteristic pressure frequencies are in good agreement, Fig. 14. In Figs. 13 and 14 it is possible to identify three main coherent structures present in the interrogated domain. In particular, the shear layer eddies that define the early separation of the flow (mode 4, Fig. 13), appear to be small and characterized by a relatively high frequency $\left(F^{+}=3\right)$. On the other hand, the larger eddies captured by mode 2 contain most of the flow's energy and travel downstream with a lower frequency $\left(F^{+}=0.7\right)$. Mode 3 bridges mode 2 and mode 4 describing the evolution of the early shear layer instability (mode 4) into larger structures (mode 2). This analysis highlights three main flow frequencies, later used to define $f_{a}$ in Eq. 26.

\subsection{5. $C_{d}$ values}

Last, a grid independence study is performed to corroborate the prediction agreement of the PANS method. Table 3 lists the coefficients of drag $C_{d}$ for different meshes and methods, while Fig. 15 shows the time histories of $C_{d}$ s for different calculations. Taking the fine LES $C_{d}$ as baseline value 

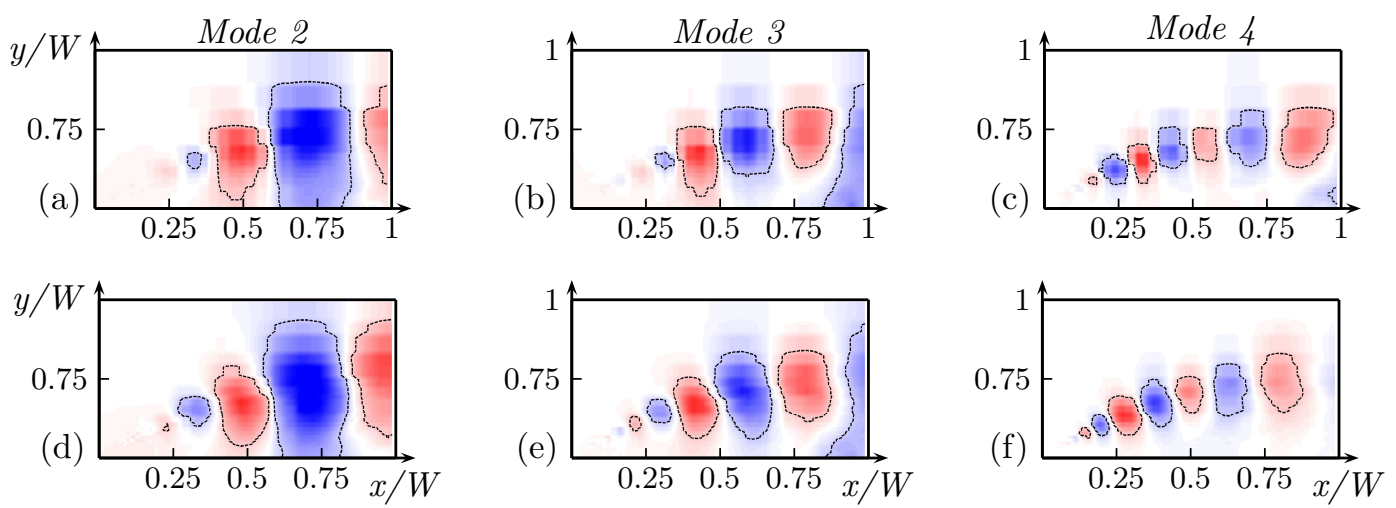

Figure 13: POD pressure modes. Comparison between coarse PANS (a-c) and resolved LES (d-f). Refer to Fig. 3 (a) for the observed domain location.
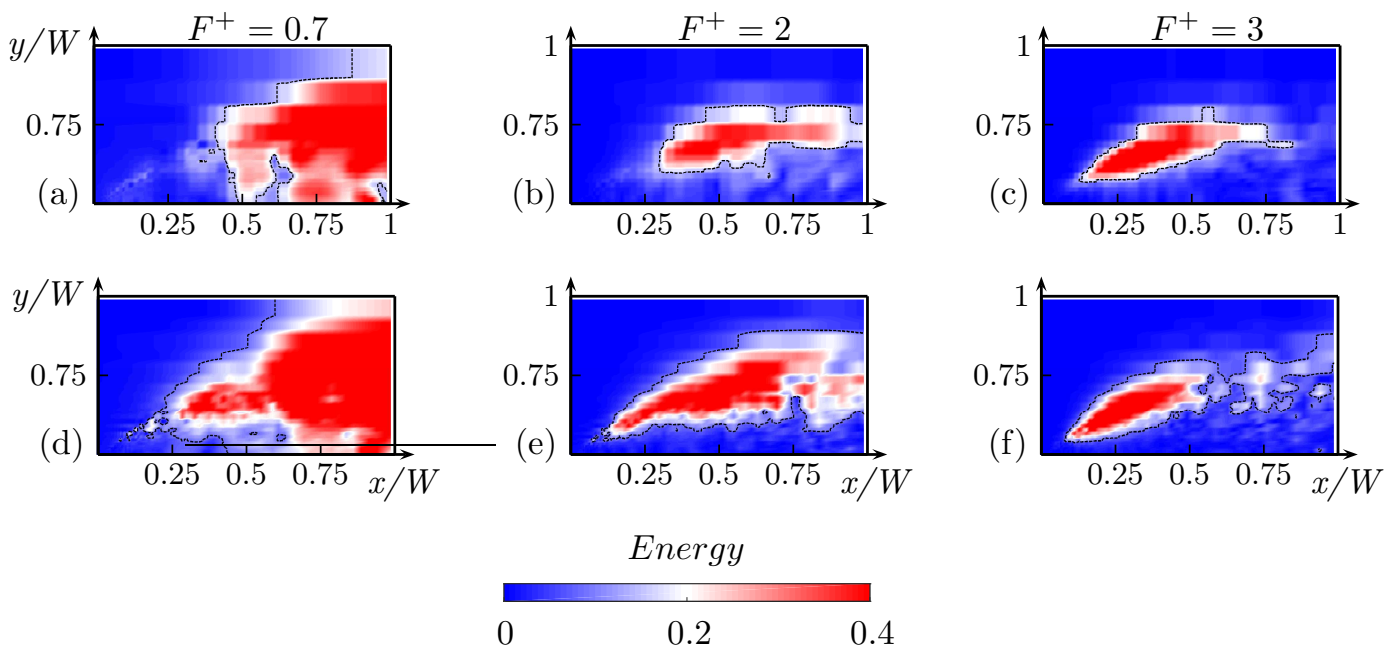

Figure 14: Spatial distribution of the energy of the pressure characteristic frequencies. The values are normalized by the maximum value of the spatially averaged spectrum. Comparison between coarse PANS (a-c) and resolved LES (d-f). Refer to Fig. 3 (a) for the observed domain location. 


\begin{tabular}{ll}
\hline Grid & $C_{d}$ \\
\hline Fine LES (16 mil.) & 1.13 \\
Medium LES (12 mil.) & 1.09 \\
Coarse LES (4 mil.) & 0.96 \\
Medium PANS ( 7 mil.) & 1.14 \\
Coarse PANS (4 mil.) & 1.08 \\
\hline
\end{tabular}

Table 3: $C_{d}$ values of LES and PANS simulations.

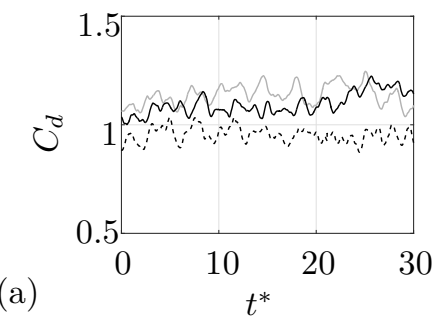

(b)

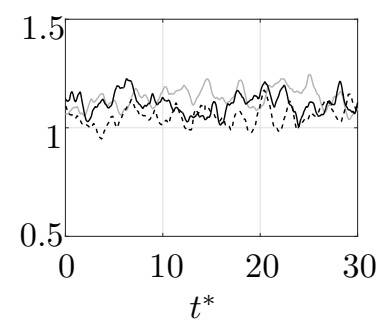

Figure 15: LES $C_{d}$ time history (a); medium LES grid (solid black line) and coarse LES grid (dashed black line). PANS $C_{d}$ time history (b); medium PANS grid (solid black line) and coarse PANS grid (dashed black line). The solid gray lines represent the baseline that is the fine LES calculation. Refer to Tab. 3 for grid sizes.

(gray solid lines in Fig. 15), the coarse LES calculation suffers a 16\% drop of $C_{d}$. In contrast, PANS holds on (within a $4 \%$ error) to the baseline value. The experimental set-up did not allow direct measurements of the aerodynamic forces, however, a further comparison between the experimental and numerical $C_{p}$ integrated values along the front and rear horizontal profiles (at $z=0$ ) of the model is presented in Tab. 4. In this case, the coarse PANS calculation is again within a $4 \%$ error when compared to LES and within a $7 \%$ error when compared to the experimental data, while the coarse LES results are $8 \%$ and $11 \%$ compared with the fine LES results and the experimental data, respectively.

\subsection{Qualitative PANS simulations of the actuated flow}

The ultimate goal of the actuation is to suppress the separation that occurs at the sides of the model. In this section a qualitative study of the AFC application is proposed. Future investigations aim to compare in a quantitative way the effects of the applied synthetic jets. Only one Reynolds number $\left(R e=5 \times 10^{5}\right)$ was simulated here. Nevertheless, the results presented in 


\begin{tabular}{ll}
\hline Case & $C_{p}$ integration \\
\hline Experiments & 1.24 \\
Fine LES (16 mil.) & 1.20 \\
Coarse LES (4 mil.) & 1.11 \\
Coarse PANS (4 mil.) & 1.16 \\
\hline
\end{tabular}

Table 4: $C_{d}$ values calculated by $C_{p}$ integration around the middle horizontal section of the model's surface $(z=0)$. Comparison between experiment and simulations.

[28], for a five times lower $R e$, are taken as a guideline (and are shown to be scalable for this $R e$ range) to design the actuation parameters used here. Moreover, previous studies $[45,22]$ have also shown little influence of the $R e$ when the orientation of the actuation is kept constant and $R e>2.5 \times 10^{5}$. However, in order to ensure the scalability of the actuation parameters, an experimental campaign on a full-scale truck model at one order of magnitude higher $R e$ is necessary and it will be conducted in the future. Figure 16 (a) and (b) show the $C_{d}$ time history and their FFT plots, respectively, for the unactuated (gray line) and the actuated (black line) cases. The mean value of $C_{d}$ is strongly related to the dimension of the recirculation bubble, Fig. 17. Controlling the flow with the shear layer frequency (mode 4 of the unforced flow, Fig. 13) the highest decrease of $C_{d}$ is observed. Moreover, moving from $F^{+}=0.7$ to $F^{+}=3$, the separated region progressively decreases, Fig. 17 . In particular, the reattachment point travel closer to the rounded corner, therefore the length and the height of the recirculation bubble is substantially reduced. The $C_{d}$ root mean square (RMS) value and the integral level of energy of $C_{d}$ are reported in Tab. 5. The actuation introduces artificial fluctuations that, for case $F^{+}=0.7$, increase the integral level of energy of the $C_{d}$ 's FFT with respect to the unactuated $C_{d}$ 's FFT signal, Tab. 5. In case $F^{+}=3$ instead, the integral level of energy of the $C_{d}$ and its RMS are drastically reduced. Figure 16 (b) shows the energy of each frequency describing $C_{d}$, normalized by the maximum value of the unactuated $C_{d}$ 's FFT. The lowest peak induced by the actuation is observed for case $F^{+}=3$. Thus, case $F^{+}=3$ introduces the least of the fluctuations in the surrounding flow field. This behaviour can also be seen by looking at the structures developed by the three actuation frequencies. Figure 18 shows the spatial distribution of the structures induced by the actuation and the strength of their periodicity over time. Figure 18 (a) shows the most energetic pressure 
POD mode of each actuated configuration. The structures formed by the first actuated case, $F^{+}=0.7$ (Fig. 18 (a)), are the largest, spreading over large part of the observed domain. On the other hand, the structures developed by the last actuated case, $F^{+}=3$ (Fig. 18 (c)), are limited to a small area of the observed domain, having less influence on $C_{d}$. In other words, the alternated, high-low pressure pattern of the first two actuated cases develops downstream affecting periodically the base region, while the third case's structures vanish or, better put, weaken before reaching the base region, Fig. 18. This explains why the $C_{d}$ fluctuations are strongly related to the dimension of the side structures. In addition, Fig. 18 also corroborates the link between structures and corresponding frequencies identified in the unactuated flow. In particular, actuation $F^{+}=0.7$ generates structures (Fig. 18 (a)) comparable to the first mode of the unactuated flow (Fig. 13 (a and d)), actuation $F^{+}=2$ generates structures (Fig. 18 (b)) comparable to the second mode of the unactuated flow (Fig. 13 (b and e)), and actuation $F^{+}=3$ generates structures (Fig. 18 (c)) comparable to the third mode of the unactuated flow (Fig. 13 (c and f)). Figure 18 (d-f) shows the orbit plot of the the temporal coefficients related to the corresponding POD mode presented in Fig. 18 (a-c). In particular, the orbit plot describes the time history of the temporal coefficients and highlights their possible periodicity. The more regular spiral the more periodic is a certain train of structures. Therefore, a strong periodicity, is observed for all three actuated cases according to their specific forcing frequency.

Figure 19 shows the different formation of the unactuated and actuated $\left(F^{+}=3\right)$ structures. The well-organized shear layer of the unactuated case changes drastically when the actuation interacts with the flow. In particular, the vortex core of the unactuated case develops evenly along the A-pillar. In contrast, the difference in upward (wall normal) flowing velocity, induced by small and periodic disturbance of the actuation, favours the formation of smaller and less organized hairpin like vortices all along the A-pillar. This behaviour is not the same for the three actuation frequencies. Figure 20 depicts the phase averaged flow field projected on the observed domain (b, d and $\mathrm{f}$ ) and four instantaneous pictures of isosurfaces of $Q$-criterion captured at four stages of the respective actuation cycle (c, e and g). Figures 20 (b and $\mathrm{d}$ ) show the presence of a clear and regular train of vortices, while case $F^{+}=3$, depicted in Fig. 20 (f), shows a more steady recirculation bubble, that does not clearly reveal the presence of a periodic pattern. Taking a closer look at the 3D structures in Fig. 21, the formation of hairpin vortices 


\begin{tabular}{llll}
\hline Case & $\overline{C_{d}}$ & $C_{d}$ RMS & $C_{d}$ Int. energy \\
\hline Unactuated & 1.08 & 0.048 & 5.01 \\
$F^{+}=0.7$ & 0.84 & 0.046 & 8.71 \\
$F^{+}=2$ & 0.78 & 0.034 & 4.20 \\
$F^{+}=3$ & 0.75 & 0.022 & 1.74 \\
\hline
\end{tabular}

Table 5: Time averaged $C_{d}$, its RMS and the integral level of energy of its FFT.

is rarely observable for case $F^{+}=0.7$. Rather, the separation of the flow is typically defined by elongated cores that span the height of the model ( $A$ in Fig. 21 and Fig. $20(\mathrm{c})$ ). Case $F^{+}=2$ visualizes a regular formation of a large hairpin structure, starting at the flow separation point and developing in the stream-wise direction ( $B$ in Fig. 21 and Fig. 20 (e)). This behaviour is also corroborated by Fig. 18, where the POD analysis shows a strong structure periodicity. The last case, $F^{+}=3$, shows the early formation of several smaller and less organized hairpin vortices $(C$ in Fig. 21 and Fig. 20 (g)). Moreover, these structures develop and dissipate soon enough to avoid the formation of a larger and organized recirculation bubble. This behaviour was also observed in previous works $[45,46]$ where was demonstrated that higher actuation frequencies, still in the receptive band of frequencies of the unactuated flow, generate structures that decay faster than structures formed by lower forcing frequencies. As a consequence, lower actuation frequencies produce strong unsteady loads, as it is also shown by the $C_{d}$ analysis, Fig. 16 and Tab. 5.

\section{Conclusions}

PANS simulations, at $R e=5 \times 10^{5}$, were conducted to analyse an active flow control strategy for a generic truck cabin. The truck cabin model is characterized by a sharp edge separation on top and bottom edges and pressure induced separation at the rounded vertical front corners (with $R / W=0.05$ ), the A-pillars. The truck cabin model was designed to put a spotlight on the A-pillar flow separation. The PANS approach was validated against experiments and resolved LES, showing the potential of capturing the main flow features, when a mesh, far from being resolved for LES, was employed. In particular, a fine grid of 16 million elements was used to compute the resolved LES, while a much coarser grid of 4 million elements was employed 

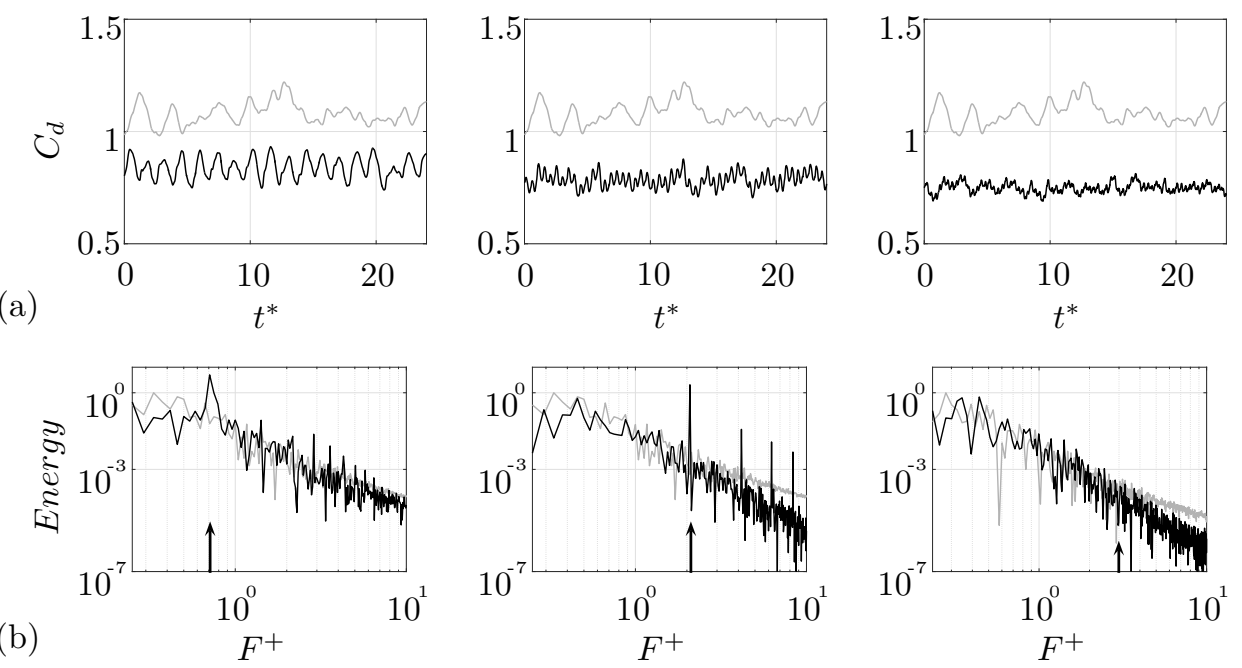

Figure 16: $C_{d}$ time history (a) and their FFT (b) of three actuated cases (black lines). (b) FFT of the $C_{d}$ signal for the unactuated and three different actuated cases. From left to right: case actuated at $F^{+}=0.7, F^{+}=2$ and $F^{+}=3$. The unactuated case (gray lines) is used as baseline. The arrows indicate the actuation frequency. The spectra are normalized by the maximum value of the unactuated spectrum.

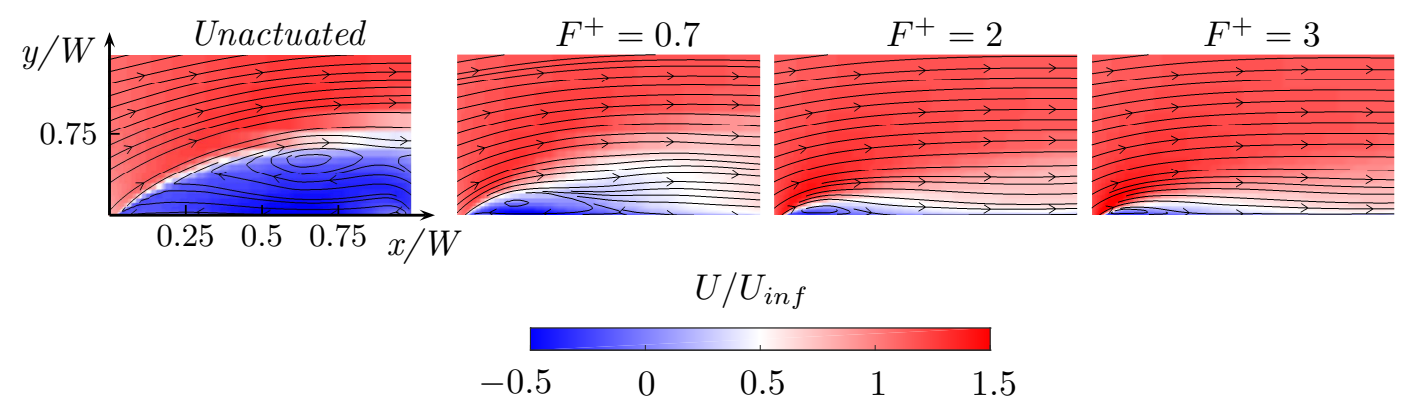

Figure 17: Averaged stream-wise velocity of the unactuated and three actuated cases. Flow from left to right. Refer to Fig. 3 (a) for the observed domain location. 

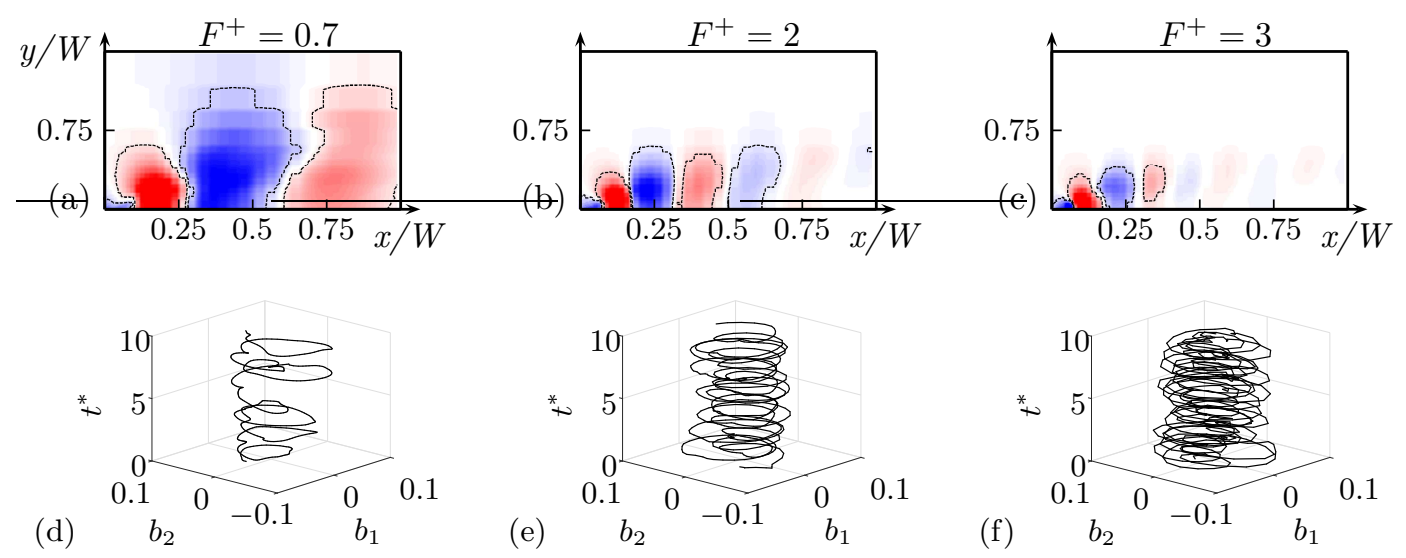

Figure 18: Most energetic POD pressure mode of three actuated cases (a-c). Orbit plots of the corresponding temporal coefficients (d-f). Cases actuated at $F^{+}=0.7$ (a and d), $F^{+}=2\left(\mathrm{~b}\right.$ and e) and $F^{+}=3(\mathrm{c}$ and $\mathrm{f}$ ). Refer to Fig. 3 (a) for the observed domain location.

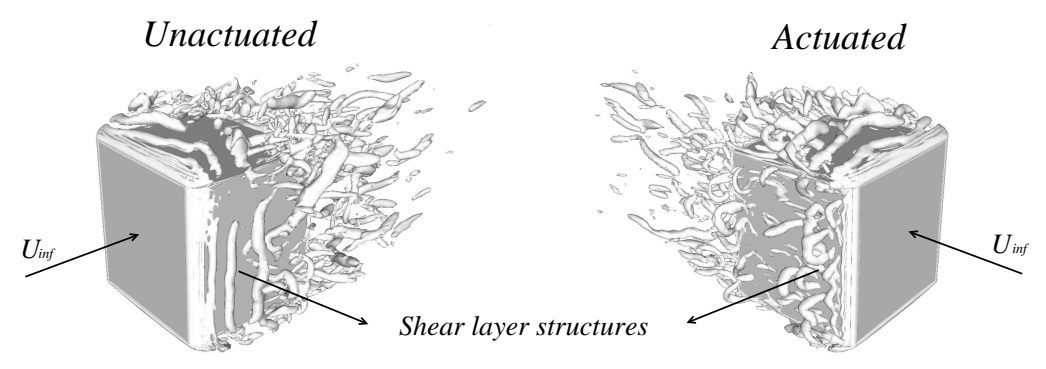

Figure 19: Isosurfaces of $Q$-criterion $\left(Q=1.5 \times 10^{5} s^{-2}\right)$. Unactuated (left) and actuated at $F^{+}=3$ (right) case. 
(a)
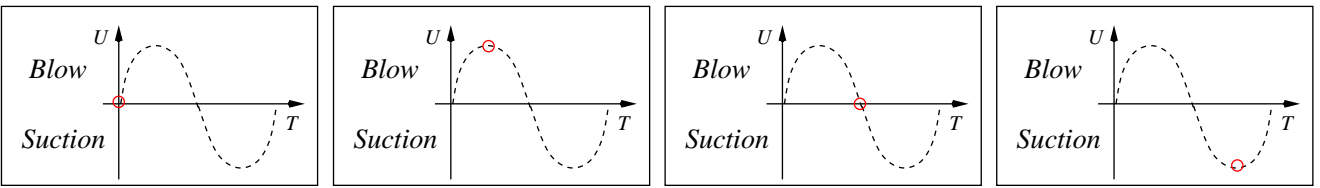

(b)
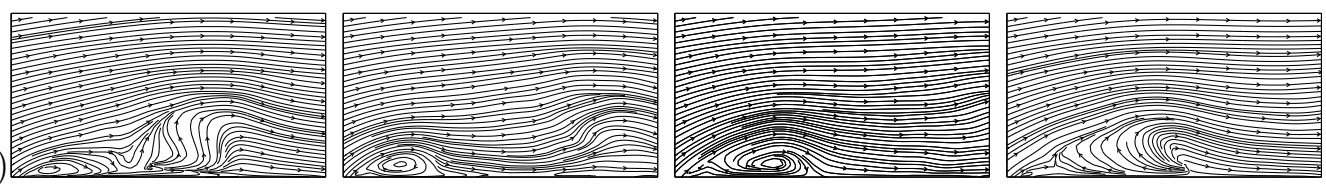

(c)
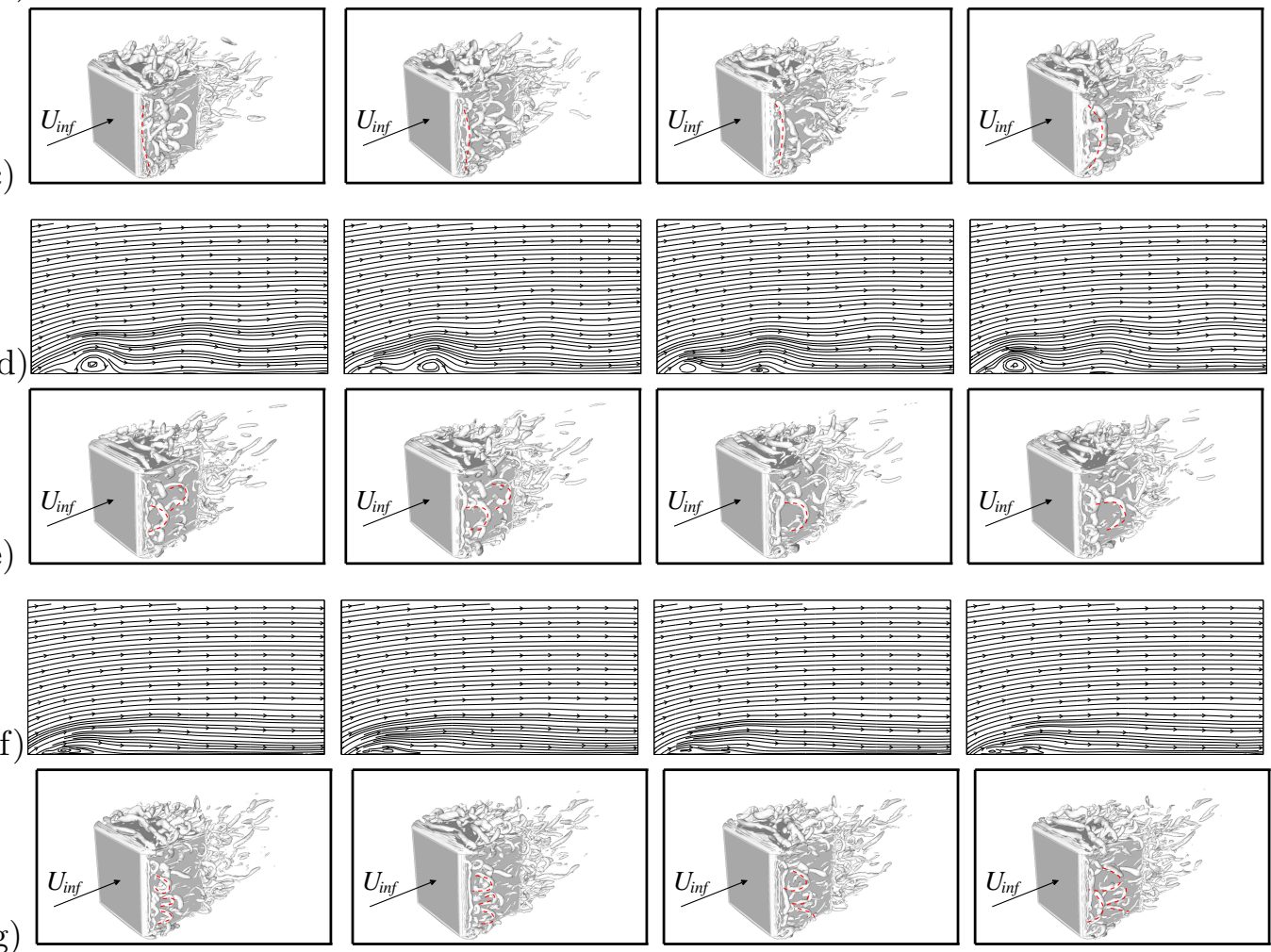

Figure 20: One actuation cycle; the red circle indicates the position of the phase average (a). Sequence of phase averaged velocity streamlines during a cycle of the actuation (b, $\mathrm{d}$ and $\mathrm{f})$. Isosurfaces of $Q$-criterion $\left(Q=1.5 \times 10^{5} \mathrm{~s}^{-2}\right)$ at four different instants of the actuation cycle (c, e and g). Flow from left to right. (b-c) $F^{+}=0.7$. (d-e) $F^{+}=2$. (d-e) $F^{+}=3$. The red dashed line indicate the vortical structures formed by different forcing frequencies. 

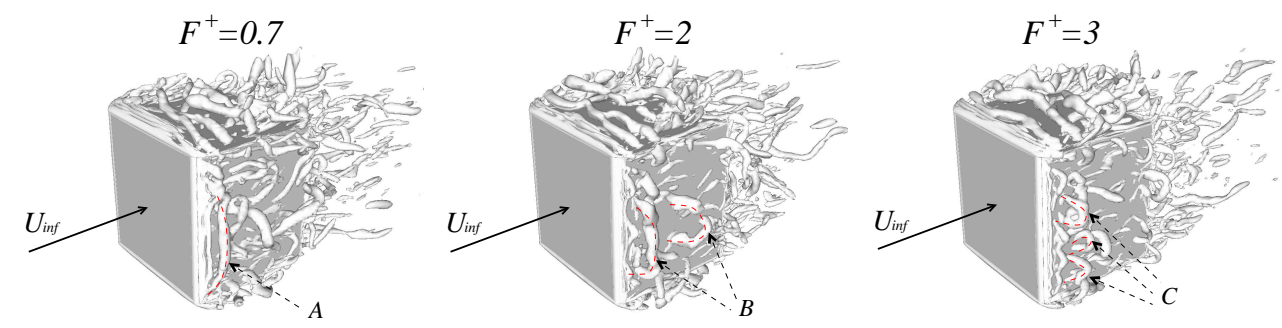

Figure 21: Isosurfaces of $Q$-criterion $\left(Q=1.5 \times 10^{5} s^{-2}\right)$. From left to right: $F^{+}=0.7$, $F^{+}=2, F^{+}=3$. The dashed red lines indicate the hairpin vortices formed by the actuation. The red dashed line indicate the vortical structures formed by different forcing frequencies.

to conduct the PANS calculations. The surface pressure profiles of $\beta=0^{\circ}$ and $\beta=10^{\circ}$ configurations are compared showing a good PANS prediction. The averaged flow velocity and stress are also compared in the observed domain region. Furthermore, the validation involved modal and frequency analyses by means of POD and FFT, respectively. The span-wise ( $y$ direction) velocity component modes produced by PANS are comparable with both experiments and LES results. The areas of interest of the characteristic frequencies of the unactuated flow are also well predicted as observed in the FFT plots. The pressure field, sampled in numerical simulations only, was further compared between PANS and LES showing a good agreement by the structures and frequencies observed in the POD and FFT analysis. The last part of the validation analysed the $C_{d}$ results from several computational grids and a comparison (PANS and LES results and experimental data) of the integrated $C_{p}$ values along an horizontal surface section of the model. Overall, the validation demonstrates a better prediction by PANS when a drastically coarsen grid is used, and a good prediction of the main important structures and frequencies of the flow field. After this process, the main frequencies and POD modes are individuated for the unactuated case. Thus, the frequencies describing the first three most energetic pressure POD modes were used to actuate the flow. This second part of the study remains qualitative since no comparison with experimental data was performed. In particular further investigation will use experiments to validate and model the correct boundary condition for a high fidelity simulation of the AFC. Nevertheless, when the actuation frequency was the one describing the shear layer instability, the highest drag reduction, a suppression of the separation bubble, and the lowest induced artificial fluctuations are observed. In addi- 
tion, the structures generated by different actuation frequencies are found to be substantially different. A low actuation frequency forms structures that have a uniform elongated vortex core along the A-pillar. In contrast, the disturbances of higher actuation frequencies form smaller and less organized hairpin like vortical structures. To summarize, an extended validation of PANS is carried out and the effects of an AFC on a heavily separated turbulent flow are qualitatively investigated. A deeper investigation is needed to verify the scalability of the actuation parameters for higher Reynolds numbers. Finally, the findings of this paper provide additional support for the conclusions drawn in previous studies $[28,29]$ and a solid foundation toward an AFC implementation for a real truck configuration.

\section{Acknowledgements}

This work is funded by the Swedish Energy Agency and supported by Volvo Trucks. Software licenses were provided by AVL List GMBH. Computations were performed at SNIC (the Swedish National Infrastructure for Computing) at the National Supercomputer Center (NSC) at LiU.

\section{References}

[1] T. C. Schuetz, Aerodynamics of Road Vehicles, Fifth Edition, SAE International, 2015.

[2] A. Roshko, Perspectives on bluff body aerodynamics, Journal of Wind Engineering and Industrial Aerodynamics 49 (1993) 79-100.

[3] H. Choi, J. Lee, H. Park, Aerodynamics of Heavy Vehicles, Annual Review of Fluid Mechanics 46 (2014) 441-468.

[4] A. Gilhaus, THE INFLUENCE OF CAB SHAPE ON AIR DRAG OF TRUCKS *, Wind Engineering and Industrial Aerodynamics 9 (1981) $77-87$.

[5] S. R. Ahmed, R. G. Gawthorpe, P. A. Mackrodt, Aerodynamics of Road and Rail Vehicles, Vehicle System Dynamics 14 (1985) 319-392.

[6] V. J. Modi, S. S. Hill, T. Yokomizo, Drag reduction of trucks through boundary-layer control, Journal of Wind Engineering and Industrial Aerodynamics 54-55 (1995) 583-594. 
[7] K. Cooper, Truck Aerodynamics Reborn - Lessons from the Past, SAE Technical Paper Series (2003).

[8] J. J. Kim, S. Lee, M. Kim, D. You, S. J. Lee, Salient drag reduction of a heavy vehicle using modified cab-roof fairings, Journal of Wind Engineering and Industrial Aerodynamics 164 (2017) 138-151.

[9] S. L. Brunton, B. R. Noack, Closed-Loop Turbulence Control: Progress and Challenges, Applied Mechanics Reviews 67 (2015).

[10] A. Brunn, W. Nitsche, Active control of turbulent separated flows over slanted surfaces, International Journal of Heat and Fluid Flow 27 (2006) 748-755.

[11] M. Ben Chiekh, M. Ferchichi, M. Michard, M. S. Guellouz, J. C. Béra, Synthetic jet actuation strategies for momentumless trailing edge wake, Journal of Wind Engineering and Industrial Aerodynamics 113 (2013) 59-70.

[12] D. Barros, J. Borée, B. R. Noack, A. Spohn, T. Ruiz, Bluff body drag manipulation using pulsed jets and Coanda effect, Journal of Fluid Mechanics 805 (2016) 422-459.

[13] J.-Z. Wu, X.-Y. Lu, A. G. Denny, M. Fan, J.-M. Wu, Post-stall flow control on an airfoil by local unsteady forcing, Journal of Fluid Mechanics 371 (1998) 21-58.

[14] A. Glezer, M. Amitay, A. M. Honohan, Aspects of Low- and HighFrequency Actuation for Aerodynamic Flow Control, AIAA Journal 43 (2005) 1501-1511.

[15] M. Amitay, A. Glezer, Role of Actuation Frequency in Controlled Flow Reattachment over a Stalled Airfoil, AIAA Journal 40 (2002) 209-216.

[16] M. Gad-el Hak, A. Pollard, J. P. Bonnet, Flow Control: Fundamentals and Practices, number v. 53 in Flow Control: Fundamentals and Practices, Springer-Verlag, 1998.

[17] D. Greenblatt, I. J. Wygnanski, Control of flow separation by periodic excitation, Progress in Aerospace Sciences 36 (2000) 487-545. 
[18] M. Amitay, F. Cannelle, Evolution of finite span synthetic jets, Physics of Fluids 18 (2006).

[19] L. N. Cattafesta, M. Sheplak, Actuators for Active Flow Control, Annual Review of Fluid Mechanics 43 (2011) 247-272.

[20] L. W. Sigurdson, A. Roshko, The Structure and Control of a Turbulent Reattaching Flow, Turbulence Management and Relaminarisation (1988) 497-514.

[21] K. Sasaki, M. Kiya, Three-Dimensional Vortex Structure in a LeadingEdge Separation Bubble at Moderate Reynolds Numbers, ASME Journal of Fluids Engineering 113 (1991) 405-410.

[22] L. Gad, S. Avraham, Flow Control Applied to the Front Rounded Edge of a Bluff Body., International Journal of Flow Control 6 (2014) 21-42.

[23] G. Lubinsky, A. Seifert, Suction and Oscillatory Blowing Applied to the Rounded Front Edges of a Square Prism BT - Instability and Control of Massively Separated Flows: Proceedings of the International Conference on Instability and Control of Massively Separated Flows, held in P, Springer International Publishing, Cham, 2015, pp. 157-162.

[24] J. A. Vernet, R. Örlü, P. H. Alfredsson, Separation control by means of plasma actuation on a half cylinder approached by a turbulent boundary layer, Journal of Wind Engineering and Industrial Aerodynamics 145 (2015) 318-326.

[25] S. Krajnović, R. Lárusson, B. Basara, Superiority of PANS compared to LES in predicting a rudimentary landing gear flow with affordable meshes, International Journal of Heat and Fluid Flow 37 (2012) 109122 .

[26] S. Krajnović, G. Minelli, B. Basara, Partially-averaged Navier-Stokes simulations of two bluff body flows, Applied Mathematics and Computation 272 (2016) 692-706.

[27] S. Krajnović, G. Minelli, B. Basara, Partially-Averaged Navier-Stokes Simulations of Flows Around Generic Vehicle at Yaw, SAE Technical Paper (2016). 
[28] G. Minelli, S. Krajnović, B. Basara, B. R. Noack, Numerical Investigation of Active Flow Control Around a Generic Truck A-Pillar, Flow, Turbulence and Combustion 97 (2016) 1235-1254.

[29] G. Minelli, E. A. Hartono, V. Chernoray, L. Hjelm, S. Krajnović, Aerodynamic flow control for a generic truck cabin using synthetic jets, Journal of Wind Engineering and Industrial Aerodynamics 168 (2017) 81-90.

[30] J. Smagorinsky, General circulation experiments with the primitive equations, Monthly weather review 91 (3) (1963) 99-165.

[31] S. Krajnović, Large eddy simulation of flows around ground vehicles and other bluff bodies., Philosophical transactions. Series A, Mathematical, physical, and engineering sciences 367 (2009) 2917-2930.

[32] S. S. Girimaji, Partially-Averaged Navier-Stokes Model for Turbulence: A Reynolds-Averaged Navier-Stokes to Direct Numerical Simulation Bridging Method, Journal of Applied Mechanics 73 (2006) 413.

[33] S. S. Girimaji, E. Jeong, R. Srinivasan, Partially Averaged NavierStokes Method for Turbulence: Fixed Point Analysis and Comparison With Unsteady Partially Averaged Navier-Stokes, Journal of Applied Mechanics 73 (2006) 422.

[34] M. Germano, Turbulence: the filtering approach, Journal of Fluid Mechanics 238 (1992) 325-336.

[35] J. M. Ma, S. H. Peng, L. Davidson, F. J. Wang, A low Reynolds number variant of partially-averaged Navier-Stokes model for turbulence, International Journal of Heat and Fluid Flow 32 (2011) 652-669.

[36] B. Basara, S. Krajnović, S. Girimaji, PANS methodology applied to elliptic-relaxation based eddy viscosity transport model, Turbulence and Interactions (2010) 63-69.

[37] B. Basara, S. Krajnovic, S. Girimaji, Z. Pavlovic, Near-Wall Formulation of the Partially Averaged Navier Stokes Turbulence Model, AIAA Journal 49 (2011) 2627-2636.

[38] S. Girimaji, K. Abdol-Hamid, Partially Averaged NavierStokes Model for Turbulence: Implementation and Validation, AIAA paper (2005). 
[39] AVL, Fire manual v2014 (2014).

[40] S. B. Pope, Turbulent Flows, 2001.

[41] U. Piomelli, J. Chasnov, Large-eddy simulations: theory and applications, Kluwer Academic Publisher, 1996.

[42] J. L. Lumley, Stochastic Tools in Turbulence - Applied Mathematics and Mechanics, Academic Press, New York, 1970.

[43] L. Sirovich, Turbulence and the dynamics of coherent structures part i: coherent structures, Quarterly of Applied Mathematics XLV (1987) $561-571$.

[44] D. Rempfer, H. F. Fasel, Evolution of three-dimensional coherent structures in a flat-plate boundary layer, Journal of Fluid Mechanics 260 (1994) 351.

[45] A. Seifert, A. Darabi, I. Wyganski, Delay of airfoil stall by periodic excitation, Journal of Aircraft 33 (1996) 691-698.

[46] L. Pack, A. Seifert, Dynamics of active separation control at high Reynolds numbers, 38th AIAA Aerospace Sciences Meeting and Exhibit (2000). 